\title{
Pathogenic mechanisms of lung adenocarcinoma in smokers and non-smokers determined by gene expression interrogation
}

\author{
YUNQIAN HU $^{1}$ and GUOHAN CHEN ${ }^{2}$ \\ Departments of ${ }^{1}$ Respiration and ${ }^{2}$ Thoracic Surgery, East Hospital, Tongji University School of Medicine, \\ Shanghai 200120, P.R. China
}

Received February 2, 2015; Accepted March 19, 2015

DOI: $10.3892 / \mathrm{ol} .2015 .3462$

\begin{abstract}
Cigarette smoking is the leading risk factor for lung cancer, which accounts for the highest number of cancer-related mortalities worldwide in men and women. Individuals with a history of smoking are 15-30 times more likely to develop lung cancer compared with those who do not smoke. However, our understanding of the underlying molecular mechanisms that contribute to lung tumorigenesis in smokers versus non-smokers remains incomplete. In order to investigate such mechanisms, the present study aimed to systemically interrogate microarray datasets from tumor biopsies and matching normal tissues from stage I and II lung adenocarcinoma patients who had never smoked or were current smokers. The gene expression analysis identified 422 (99 upregulated and 323 downregulated) and 534 (174 upregulated and 360 downregulated) differentially-expressed genes from the never-smokers and current smokers, respectively, and the two groups shared 277 genes that exhibited similar trends of alteration. These genes encode regulators that are involved in a variety of cellular functions, including collagen metabolism and homeostasis of caveolae plasma membranes. Gene Ontology and Kyoto Encyclopedia of Genes and Genomes characterization indicated that biological pathways, including extracellular matrix-receptor interaction and cell migration and proliferation, were all affected in the lung cancer patients regardless of the smoking status. However, smoking induced a unique gene expression pattern characterized by upregulation of cell cycle regulators $(C D K 1, C C N B 1$ and $C D C 20)$, as well as significantly affected biological networks, including p53 signaling pathways. Taken together, these findings suggest novel mechanistic insights, and provide an improved understanding of the smoking-induced molecular alterations that contribute to the pathogenesis of lung adenocarcinoma.
\end{abstract}

Correspondence to: Mr. Guohan Chen, Department of Thoracic Surgery, East Hospital, Tongji University School of Medicine, 155 Jimo Road, Shanghai 200120, P.R. China

E-mail: chghandf@163.com

Key words: lung adenocarcinoma, smoking, microarray analysis, cell proliferation, $\mathrm{p} 53$

\section{Introduction}

Lung cancer remains the leading cause of cancer-related mortality worldwide (1). In the United States this year, an estimated 224,210 individuals will be diagnosed with lung cancer, with $\sim 159,260$ fatalities anticipated to occur, as reported by the Surveillance, Epidemiology, and End Results Program at the National Cancer Institute (2). The same program also found an average 5-year survival rate of merely $16.8 \%$, thus demonstrating an extremely unfavorable prognosis for lung cancer patients. This primarily results from late-stage diagnosis and a lack of effective late-stage interventions (2). Among the various histological forms of lung cancer, non-small cell adenocarcinoma constitutes the most common subtype, and thus presents the greatest challenge for patients and caregivers.

Lung adenocarcinoma is a malignant tumor of the glandular cells, a specialized cell type that produces mucus and supports internal structural integrity. The underlying pathogenic mechanism leading to bronchial malignancy remains largely elusive. It has been established that smoking plays a significant role in the initiation and progression of lung adenocarcinoma (3-6). However, 10-40\% of cases occur in patients with no reported smoking history, suggesting the involvement of other risk factors, including environmental exposure and genetic susceptibility (7). Patients with a smoking history harbor 10-fold more frequent point mutations compared with never-smokers, as demonstrated by two elegant system-based genetic studies that utilized global whole-genome sequencing to provide convincing evidence that smoking exerts a profound effect on the overall genomic architecture $(8,9)$. Notably, the spectrum of mutated genes for smokers compared with never-smokers appears to be largely distinct. For example, multiple independent studies reported a significant association of $K R A S$ and epidermal growth factor receptor (EGFR) mutations with smokers and never-smokers, respectively (8-11), indicating different oncogene-driven mechanisms that depend upon smoking status.

Despite these promising findings, the mechanism by which the expression of cancer-relevant genes dictates the temporal and dynamic development of lung cancer, particularly in the early stages, remains to be fully characterized. To address this issue, the present study aimed to analyze global gene expression profiles from never-smoker (NS) and current smoker 
(CS) patients with stage I and II lung adenocarcinoma. This was greatly facilitated by public access to Gene Expression Omnibus (GEO; http://www.ncbi.nlm.nih.gov/geo/), an international repository that encourages the archival and retrieval of high-throughput datasets for versatile and independent investigation. Microarray expression profiles (accession number, GSE10072) that were derived from fresh frozen tumor and paired normal tissues of lung adenocarcinoma patients were extracted (12). While the original study primarily focused on pair-wise comparison between the NS and CS groups (12), the current study re-examined the expression dataset by systemically comparing tumor tissue with normal samples within either the NS or CS group. This strategy maximized the signal-to-noise ratio by reducing expression variations due to individual differences, and facilitated more effective determination of smoking-dependent and -independent molecular mechanisms involved in lung carcinogenesis. Through various data mining approaches, gene expression profiles and biological pathways that are important for initiation and development of lung cancers were identified.

\section{Materials and methods}

Data collection. A search of GEO was conducted, and one microarray expression dataset (GSE10072) (12) was downloaded. Fresh frozen tissue samples of lung adenocarcinoma and paired non-involved lung tissue were obtained from NS patients, defined as individuals who had smoked $\leq 100$ cigarettes during their lifetime, and CS patients. All patients were enrolled at East Hospital of Tongji University School of Medicine between 2012 and 2013. To exclude possible changes in gene expression due to advanced tumor status, only patients with tumor tissues at the early stages (stage I and II) were selected as research subjects. Specifically, 9 normal samples and 10 tumor samples were dissected from the NS patients, and 13 normal samples and 20 tumor samples from the CS patients. The Affymetrix Human Genome U133A Array (Affymetrix, Inc., Santa Clara, CA, USA) was used as the profiling platform. Unprocessed data (.cel files) were collected, and the probe annotation files downloaded accordingly for further investigation.

Data processing and filtering. A number of algorithms were available to quantify and integrate microarray intensity. GeneChip Robust Multichip Average (GC-RMA) in the R package gcrma (13) was selected and utilized in the present study. The normalization process was conducted in three steps: Model-based background correction, quantile normalization and summarization. In order to filter out uninformative data (control probesets, other internal controls and genes with below-background expression), the genefilter package in $\mathrm{R}$ language with nsFilter function was utilized. However, probesets without Entrez Gene identifiers or with identical Entrez Gene identifiers were not removed by the filter.

Differentially-expressed gene (DEG) analysis. Two physiologically relevant comparisons were statistically investigated. Comparison 1 was made between tumor biopsies and matching normal tissues from NS patients, whereas comparison 2 was made between these tissue types in CS patients. By means of the R package Limma (14) in Bioconductor, significantly altered gene expression was determined in tumor samples compared with normal controls. For probes that had identical Entrez Gene identifiers, only the probe exhibiting the largest variance was included for downstream DEG analysis. Genes with a $\log _{2}$ (fold change)l of $>2$, and an adjusted $P$-value of $<0.01$ were defined as significantly differentially expressed between the two groups. The adjusted P-value was calculated through Benjamini and Hochberg's false discovery rate correction on the original P-value. Significant DEGs identified in CS and NS patients were then investigated in parallel to determine genes for which the altered expression was specific to one group or common to both groups.

Validation of DEGs by reverse transcription quantitative polymerase chain reaction $(R T-q P C R)$. To validate that the expression of the identified DEGs was significantly altered, the top five genes, which were identified as those exhibiting the highest degree values, as determined by Cytoscape (15), where a higher degree indicates a higher number of connections between genes, in each comparison were re-evaluated using an independent collection of tumor biopsies and matching healthy controls. Six patients with lung adenocarcinoma were recruited from the CS or NS groups (Table I), and all participants were duly informed of the procedures and consented to the use of biological samples. The protocol and consent forms were approved by the Tongji University School of Medicine Human Subjects Committee. Tumor samples and adjacent non-involved normal tissues were collected from each subject, followed by RNA extraction using TRIzol RNA isolation reagent (Life Technologies, Grand Island, NY, USA). From each sample, $2 \mu \mathrm{g}$ RNA was transcribed into cDNA using M-MLV Reverse Transcriptase (Invitrogen Life Technologies, Carlsbad, CA, USA) following the manufacturer's instructions. cDNA was then used as a template for PCR, which was performed using SYBR green reagent (Applied Biosystems Life Technologies, Foster City, CA, USA). The total PCR reaction volume was $20 \mu \mathrm{l}(10 \mu \mathrm{l}$ 2X Master Mix, $1 \mu \mathrm{l}$ forward primer, $1 \mu \mathrm{l}$ reverse primer, $5 \mu \mathrm{ldd} \mathrm{H}_{2} \mathrm{O}$ and $\left.3 \mu \mathrm{lcDNA}\right)$. The following gene-specific primers were used: Forward, 5'-TGGAGGTGTGAACTC TTCGTC-3' and reverse, 5'-TCTGTCCGTGCTTCATAG TCA for $F Y N$; forward, 5'-TTTGCCTGAAATGGTGAGTAA GG-3' and reverse, 5'-TGGTTTGCTTGAGCTGTGTTC-3' for FLT1; forward, 5'-CCCGCCAGTCAGAAGTTGAG-3' and reverse, 5'-AGTCCCTTCGAGGAACACTTG-3' for BLNK; forward, 5'-GGGGCAAGGTGGAACAGTTAT-3' and reverse, 5'-GGGGCAAGGTGGAACAGTTAT-3' and reverse, 5'-CCG CTTGGAGTGTATCAGTCA-3' for FOS; forward, 5'-AAG GACTGGTACTATACCCACAG-3' and reverse, 5'-TGTCTG CTTGGTCTTTATCAACC-3' for EFNB2; forward, 5'-GGA TGTGCTTATGCAGGATTCC-3' and reverse, 5'-CATGTA CTGACCAGGAGGGATAG-3' for CDK1; forward, 5'-AAT AAGGCGAAGATCAACATGGC-3' and reverse, 5'-TTT GTTACCAATGTCCCCAAGAG-3' for CCNB1; forward, 5'-CGGGGGGTGAGGTACTTGGTCATAATCTGAATT TCGGCACCT-3' and reverse, 5'-CAGTAACGATGAGAG GACCCT-3' for STAT1; forward, 5'-GGAATATGCACCACT TGGAACA-3' and reverse, 5'-TAAGACAGGGCATTTGCC AAT-3' for AURKA; forward, 5'-GACCACTCCTAGCAAACC TGG-3' and reverse, 5'-GGGCGTCTGGCTGTTTTCA-3' for 
Table I. Clinical characteristics of patients recruited for the validation of differentially expressed genes.

\begin{tabular}{lccclcc}
\hline Patient & Gender & Age, years & $\begin{array}{c}\text { Smoking } \\
\text { status }\end{array}$ & Diagnosis & Localization & Stage \\
\hline 1 & Male & 68 & CS & Adenocarcinoma & Middle lobe of right lung & I \\
2 & Female & 59 & NS & Adenocarcinoma & Inferior lobe of left lung & II \\
3 & Female & 59 & CS & Adenocarcinoma & Inferior lobe of right lung & II \\
4 & Male & 49 & NS & Adenocarcinoma & Upper lobe of right lung & II \\
5 & Male & 72 & NS & Adenocarcinoma & Inferior lobe of left lung & I \\
6 & Male & 67 & CS & Adenocarcinoma & Inferior lobe of left lung & II \\
\hline
\end{tabular}

CS, current smoker; NS, never-smoker.

CDC20; forward, 5'-CATCCCGATGGCACTCATCTG-3' and reverse, 5'-TGCACTGAATCTCAATCAGGAAG-3' for CAV1; forward, 5'-GGGCAGCAGACCACTATGG-3' and reverse, 5'-CCAGGGTTGATGGCCTGAG-3' for FGR; forward, 5'-CCTCAGACGACAATGACACGG-3' and reverse, 5'-CTCGCTGGAATGCTTCGAGAT-3' for ZBTB16; forward, 5'-GCCTGTGCTGATCTGGTCAT-3' and reverse, 5'-AAT GGAAGTCCAAAACTCGCA-3' for ADRB2; and forward, 5'-TGGGCTGGAGTGTTACATTCA-3' and reverse, 5'-GGG GTGAGGTACTTGGTCATAAT-3' for GRK5. PCR was performed under the following conditions: Denaturation at $95^{\circ} \mathrm{C}$ for $50 \mathrm{sec}$, followed by 40 cycles of $95^{\circ} \mathrm{C}$ for $30 \mathrm{sec}, 58^{\circ} \mathrm{C}$ for $25 \mathrm{sec}, 72^{\circ} \mathrm{C}$ for $25 \mathrm{sec}$ and extension at $95^{\circ} \mathrm{C}$ for $30 \mathrm{sec}$, $55^{\circ} \mathrm{C}$ for $30 \mathrm{sec}$ and $95^{\circ} \mathrm{C}$ for $30 \mathrm{sec}$. Relative gene expression was normalized to $\beta$-actin and calculated using the $2-\Delta \Delta \mathrm{Ct}$ method (16). The statistical data were assessed using GraphPad Prism 5.0 software. Statistical analysis was performed using a two tailed, paired Student's $t$-test. $\mathrm{P}<0.05$ was considered to indicate a statistically significant difference.

Hierarchical clustering. Hierarchical clustering was conducted to classify analyzed samples according to DEG-based global gene expression profiles. The DEGs, which were classified in specific biological processes [Gene Ontology (GO) terms; http://www.geneontology.org] and Kyoto Encyclopedia of Genes and Genomes (KEGG; http://www.genome.jp/kegg/) pathways, were further extracted and the expression pattern of those DEGs characterized. Heat maps were generated for the DEGs classified in targeted biological processes or KEGG pathways using the $\mathrm{R}$ package gplots (17).

$G O$ and KEGG pathway analysis. Multiple $\mathrm{R}$ packages, including GO.db (18), KEGG.d (19) and KEGGREST (20), were utilized to detect GO categories and KEGG pathways with significant over-representation in DEGs compared with the whole genome. The significantly enriched biological processes were identified as those with $\mathrm{P}<0.01$, while for KEGG pathways, $\mathrm{P}<0.05$ was set as the threshold value.

Construction of biological network. Protein-protein interaction (PPI) pairs were downloaded from the Human Protein Reference Database (HPRD; http://www.hprd.org/), Biological General Repository for Interaction Datasets
(BioGRID; http://thebiogrid.org/) and Human Protein-protein Interaction Prediction (PIP; http://www.compbio.dundee. ac.uk/www-pips/index.jsp) to estimate and analyze interactions between genes. As a result, 561,405 pair interactions were included in the present database, and an interaction network was constructed using Cytoscape (15). Interacted gene pairs included in the curated PPI database were imported as stored networks. Following functional enrichment analysis, the DEGs specified in markedly altered biological processes (GO terms) and KEGG pathways were mapped to corresponding networks in order to systemically analyze interactions.

\section{Results}

Differential expression analysis. Pair-wise comparison was performed between lung tumor tissues and adjacent non-involved normal controls within NS or CS patients, in order to identify DEGs characterized by $\mid \log _{2}$ (fold-change) $\mid>2$ and adjusted P-value of $<0.01$. A total of 523 and 691 probes were found to be significantly altered for the NS and CS groups, accounting for 422 (99 upregulated and 323 downregulated) and 534 (174 upregulated and 360 downregulated) DEGs, respectively (Fig. 1A). Among these identified DEGs, 277 altered genes were shared by the CS and NS populations (Fig. 1B), indicating similar genetic mechanisms of lung adenocarcinoma that are likely to be independent of smoking status. However, 257 DEGs were identified in the CS group compared with 145 in the NS group (Fig. 1B), indicating that smoking induces prominent molecular alterations that contribute to the early stages of lung cancer.

Expression validation of DEGs. To verify the results of the DEG analysis, each of the top five genes that were commonly shared by the NS and CS groups (CAV1,FGR, ZBTB16, ADRB2 and GRK5), specific to the NS group (FYN, FLTl, BLNK, $F O S$ and $E F N B 2)$ or specific to the CS group (CDK1, CCNB, STAT1, AURKA and CDC20) were validated using qRT-PCR. As shown in Fig. 2, the results indicated that all these genes exhibited the same expression patterns, as identified by the differential expression analysis, thereby corroborating the validity of the microarray analysis.

Construction of PPI network. Integrative investigation that combines DEGs into biologically relevant networks may 
A

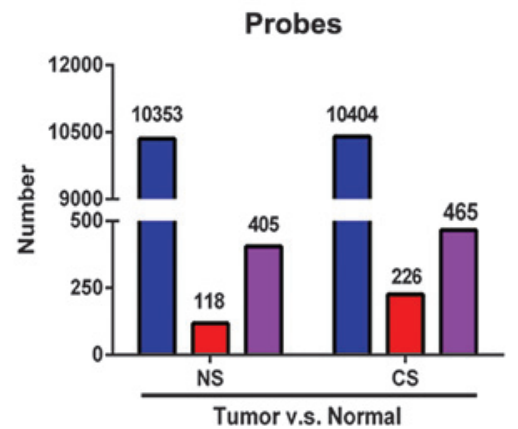

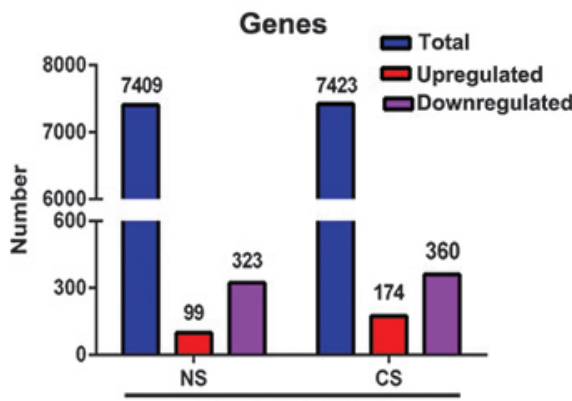

Tumor v.s. Normal

B

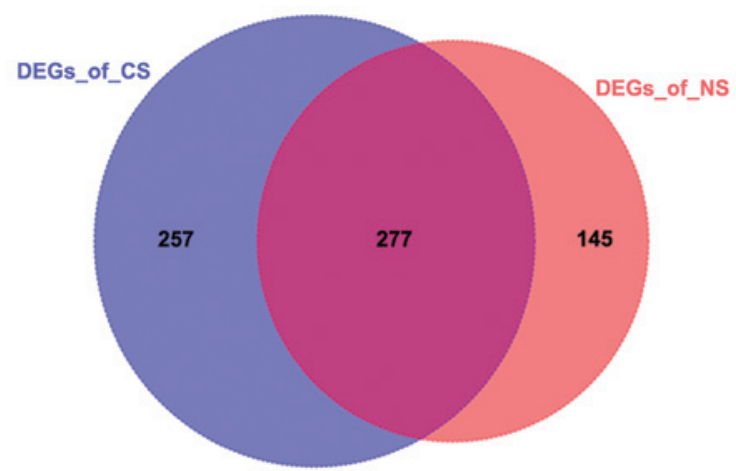

Figure 1. Identification of DEGs that were significantly correlated with lung adenocarcinoma in NS and CS patients. (A) The number of total and differentially-expressed probes and genes from the microarray GSE10072 dataset were determined for lung tumor biopsies compared with paired normal tissue controls. Blue, total number of probes or genes examined in the microarray; red, number of upregulated probes or genes; purple, number of downregulated probes or genes. (B) Venn diagram showing significant DEGs that were shared and unique for the NS and CS group. Cutoff threshold for significant DEGs was defined with llog fold-changel $>2$ and adjusted P-value of <0.01. DEG differentially-expressed gene; NS, never-smoker; CS, current smoker.
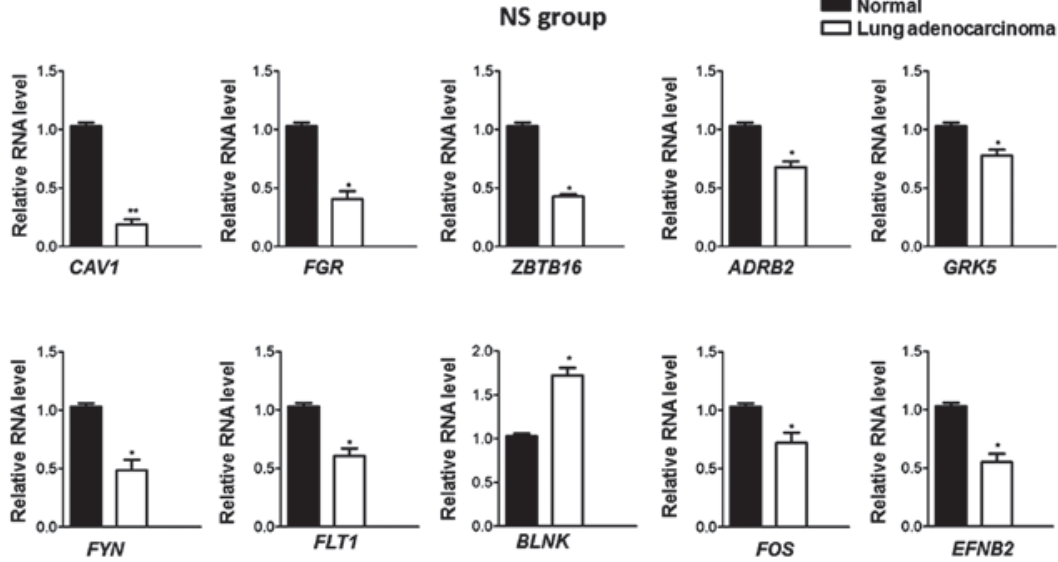

CS group
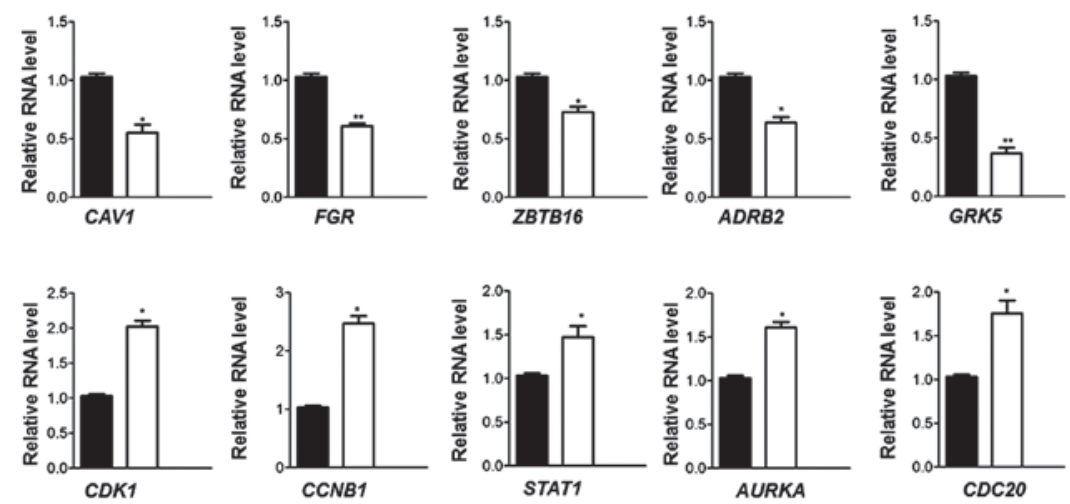

Figure 2. Validation of top five DEGs. Tumor biopsies and matching normal samples were collected from lung cancer patients from the NS or CS groups and subjected to total RNA extraction. Quantitative polymerase chain reaction was then performed for DEGs that were commonly shared by the NS and CS groups, or were specific to either the NS or CS groups. Relative RNA level was normalized to endogenous $\beta$-actin expression. All values represent the mean \pm standard error of the mean from $\mathrm{n}=6$ patients. ${ }^{*} \mathrm{P}<0.05$ and $^{* *} \mathrm{P}<0.01$ vs. normal lung samples. DEG, differentially-expressed gene; NS, never-smoker; CS, current smoker. 


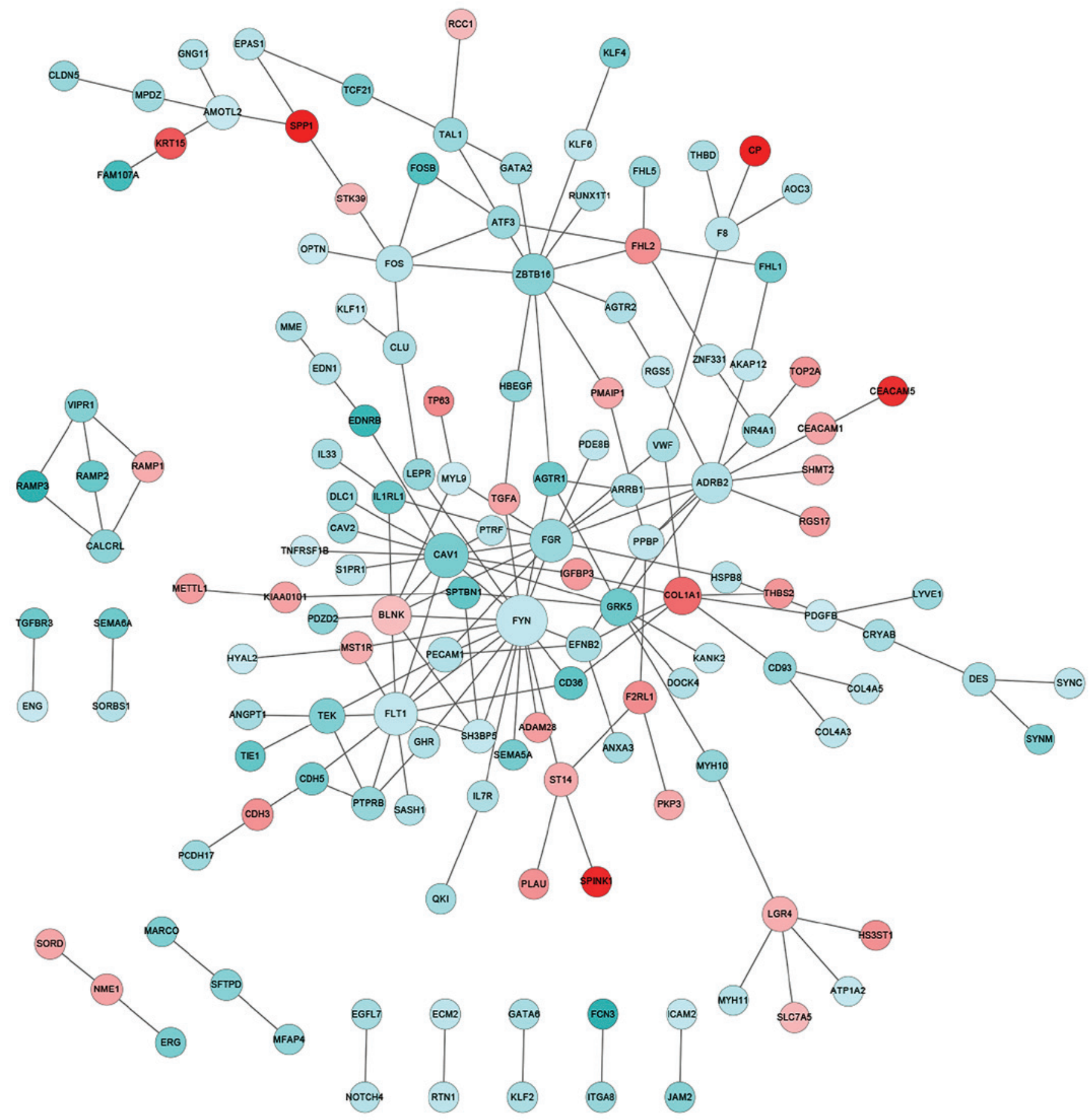

Figure 3. Interaction networks of DEGs involved in lung carcinogenesis in NS patients. Co-expression protein-protein interaction networks that comprise significant DEGs identified for the NS group. Nodes represent DEG-encoding products, with blue indicating downregulation and red indicating upregulation. DEG, differentially-expressed gene; NS, never-smoker.

provide improved mechanistic insights. Therefore, a large curated PPI database was explored to identify interactive networks that were significantly altered in stage I and II lung cancer patients. The PPI database in the present study included 561,405 pairs of interactions that were collected cumulatively from the HPRD, BioGRID and PIP databases. In the tumor biopsies from the NS patients, notable gene participants in the PPI network with significantly changed expression compared with that of normal controls included family members of protein tyrosine kinases ( $F Y N$ and
$F G R$ ), vascular endothelial growth factor (VEGF) receptors (FLT1) and zinc finger transcription factors (ZBTB16) (Fig. 3). In particular, $F Y N$ appeared to be a critical player, interacting with a total of 17 DEGs and serving as the center of the PPI network that was significantly altered (Fig. 3). By contrast, for CS patients, the key components of significantly altered PPI networks were largely involved in cell proliferation (CDK1, CCNB1, AURKA and CDC20), collagen homeostasis (COL1A1) and growth factor signaling (STAT1) (Fig. 4). Notably, the Ser/Thr protein kinase, CDK1, which 
Table II. Top 50 most significant differentially-expressed genes in the biological network implicated in lung carcinogenesis for never-smoker patients.

\begin{tabular}{|c|c|c|c|c|}
\hline Gene symbol & $\log _{2}($ fold change $)$ & P-value & Adjusted P-value & Degree \\
\hline$F Y N$ & -2.21 & $1.64 \times 10^{-5}$ & $4.07 \times 10^{-4}$ & 17 \\
\hline$C A V 1^{*}$ & -4.01 & $1.57 \times 10^{-9}$ & $4.36 \times 10^{-7}$ & 12 \\
\hline FLT1 & -2.19 & $5.43 \times 10^{-7}$ & $2.94 \times 10^{-5}$ & 11 \\
\hline$F G R^{*}$ & -3.20 & $2.69 \times 10^{-7}$ & $1.74 \times 10^{-5}$ & 11 \\
\hline ZВТВ16* & -3.62 & $1.43 \times 10^{-5}$ & $3.69 \times 10^{-4}$ & 10 \\
\hline$A D R B 2^{*}$ & -2.52 & $8.27 \times 10^{-5}$ & $1.34 \times 10^{-3}$ & 9 \\
\hline$B L N K$ & 2.09 & $1.04 \times 10^{-4}$ & $1.60 \times 10^{-3}$ & 8 \\
\hline$G R K 5^{*}$ & -4.34 & $1.82 \times 10^{-7}$ & $1.33 \times 10^{-5}$ & 7 \\
\hline COL1A1* & 4.29 & $1.57 \times 10^{-5}$ & $3.95 \times 10^{-4}$ & 7 \\
\hline FOS & -2.42 & $4.96 \times 10^{-4}$ & $5.09 \times 10^{-3}$ & 6 \\
\hline$T E K^{*}$ & -3.83 & $5.20 \times 10^{-9}$ & $9.78 \times 10^{-7}$ & 5 \\
\hline$P P B P^{*}$ & -2.21 & $9.34 \times 10^{-5}$ & $1.48 \times 10^{-3}$ & 5 \\
\hline PECAMI ${ }^{*}$ & -2.55 & $2.02 \times 10^{-9}$ & $5.34 \times 10^{-7}$ & 5 \\
\hline$L G R 4^{*}$ & 2.46 & $3.19 \times 10^{-6}$ & $1.19 \times 10^{-4}$ & 5 \\
\hline FHL2* & 3.29 & $4.04 \times 10^{-5}$ & $7.93 \times 10^{-4}$ & 5 \\
\hline EFNB2 & -2.73 & $1.45 \times 10^{-4}$ & $2.07 \times 10^{-3}$ & 5 \\
\hline$T A L 1^{*}$ & -3.22 & $2.31 \times 10^{-8}$ & $2.87 \times 10^{-6}$ & 4 \\
\hline ST14 & 2.55 & $6.68 \times 10^{-8}$ & $6.34 \times 10^{-6}$ & 4 \\
\hline SH3BP5 & -2.15 & $1.51 \times 10^{-7}$ & $1.16 \times 10^{-5}$ & 4 \\
\hline$P T P R B^{*}$ & -3.33 & $1.84 \times 10^{-4}$ & $2.46 \times 10^{-3}$ & 4 \\
\hline$F 8^{*}$ & -2.36 & $5.14 \times 10^{-4}$ & $5.20 \times 10^{-3}$ & 4 \\
\hline AMOTL2 & -2.08 & $5.61 \times 10^{-5}$ & $1.01 \times 10^{-3}$ & 4 \\
\hline$A G T R 1^{*}$ & -4.31 & $3.11 \times 10^{-10}$ & $1.38 \times 10^{-7}$ & 4 \\
\hline$V W F^{*}$ & -2.86 & $3.07 \times 10^{-8}$ & $3.58 \times 10^{-6}$ & 3 \\
\hline VIPRI $1^{*}$ & -3.73 & $8.14 \times 10^{-6}$ & $2.49 \times 10^{-4}$ & 3 \\
\hline$S P T B N 1^{*}$ & -4.57 & $1.32 \times 10^{-10}$ & $7.45 \times 10^{-8}$ & 3 \\
\hline$S P P 1^{*}$ & 7.14 & $1.01 \times 10^{-10}$ & $6.60 \times 10^{-8}$ & 3 \\
\hline$N R 4 A I^{*}$ & -2.81 & $5.04 \times 10^{-4}$ & $5.14 \times 10^{-3}$ & 3 \\
\hline MYL9 & -2.04 & $3.14 \times 10^{-5}$ & $6.48 \times 10^{-4}$ & 3 \\
\hline$M S T I R$ & 2.44 & $1.32 \times 10^{-7}$ & $1.07 \times 10^{-5}$ & 3 \\
\hline ILIRLI* & -4.30 & $1.49 \times 10^{-4}$ & $2.11 \times 10^{-3}$ & 3 \\
\hline$F 2 R L 1$ & 3.34 & $1.02 \times 10^{-6}$ & $5.13 \times 10^{-5}$ & 3 \\
\hline$D E S$ & -2.85 & $3.88 \times 10^{-6}$ & $1.39 \times 10^{-4}$ & 3 \\
\hline$C L U^{*}$ & -2.74 & $3.79 \times 10^{-4}$ & $4.14 \times 10^{-3}$ & 3 \\
\hline$C D H 5^{*}$ & -4.20 & $3.75 \times 10^{-10}$ & $1.54 \times 10^{-7}$ & 3 \\
\hline$C D 93^{*}$ & -3.36 & $4.01 \times 10^{-8}$ & $4.25 \times 10^{-6}$ & 3 \\
\hline$C D 36^{*}$ & -4.65 & $8.07 \times 10^{-10}$ & $2.56 \times 10^{-7}$ & 3 \\
\hline$C A L C R L^{*}$ & -3.55 & $7.65 \times 10^{-9}$ & $1.25 \times 10^{-6}$ & 3 \\
\hline$A T F 3^{*}$ & -3.23 & $1.09 \times 10^{-3}$ & $8.99 \times 10^{-3}$ & 3 \\
\hline ARRBI & -2.46 & $4.24 \times 10^{-7}$ & $2.46 \times 10^{-5}$ & 3 \\
\hline ZNF331 & -2.25 & $4.75 \times 10^{-6}$ & $1.63 \times 10^{-4}$ & 2 \\
\hline$T G F A$ & 2.70 & $1.30 \times 10^{-5}$ & $3.44 \times 10^{-4}$ & 2 \\
\hline$T C F 21^{*}$ & -4.12 & $3.99 \times 10^{-7}$ & $2.37 \times 10^{-5}$ & 2 \\
\hline STK39 & 2.22 & $8.23 \times 10^{-8}$ & $7.35 \times 10^{-6}$ & 2 \\
\hline SFTPD & -3.69 & $3.23 \times 10^{-5}$ & $6.64 \times 10^{-4}$ & 2 \\
\hline RGS5 & -2.09 & $1.02 \times 10^{-5}$ & $2.94 \times 10^{-4}$ & 2 \\
\hline$R A M P 3^{*}$ & -5.81 & $4.17 \times 10^{-10}$ & $1.60 \times 10^{-7}$ & 2 \\
\hline$R A M P 2^{*}$ & -4.42 & $8.28 \times 10^{-11}$ & $6.13 \times 10^{-8}$ & 2 \\
\hline RAMP1 & 2.51 & $1.33 \times 10^{-5}$ & $3.51 \times 10^{-4}$ & 2 \\
\hline PMAIP1 & 2.61 & $1.17 \times 10^{-3}$ & $9.47 \times 10^{-3}$ & 2 \\
\hline
\end{tabular}

*Genes also identified in the protein-protein interaction network for current smoker patients (Table II). 
Table III. Top 50 most significant differentially-expressed genes in the biological network implicated in lung carcinogenesis for current smoker patients.

\begin{tabular}{|c|c|c|c|c|}
\hline Gene symbol & $\log _{2}($ fold change $)$ & P-value & Adjusted P-value & Degree \\
\hline$C D K 1$ & 3.30 & $4.05 \times 10^{-9}$ & $1.39 \times 10^{-7}$ & 25 \\
\hline$C C N B 1$ & 4.13 & $1.26 \times 10^{-11}$ & $1.12 \times 10^{-9}$ & 17 \\
\hline STAT1 & 2.13 & $5.52 \times 10^{-6}$ & $6.15 \times 10^{-5}$ & 16 \\
\hline$A U R K A$ & 3.28 & $8.81 \times 10^{-10}$ & $4.01 \times 10^{-8}$ & 14 \\
\hline$C D C 20$ & 3.85 & $8.04 \times 10^{-9}$ & $2.49 \times 10^{-7}$ & 14 \\
\hline COLIAI* & 5.12 & $1.49 \times 10^{-10}$ & $8.91 \times 10^{-9}$ & 13 \\
\hline$C A V 1^{*}$ & -4.11 & $3.74 \times 10^{-13}$ & $6.69 \times 10^{-11}$ & 12 \\
\hline$F G R^{*}$ & -3.58 & $2.58 \times 10^{-8}$ & $6.96 \times 10^{-7}$ & 11 \\
\hline$T A L 1^{*}$ & -2.28 & $2.50 \times 10^{-8}$ & $6.76 \times 10^{-7}$ & 10 \\
\hline MCM2 & 2.94 & $1.01 \times 10^{-9}$ & $4.42 \times 10^{-8}$ & 9 \\
\hline$B U B 1$ & 2.60 & $1.58 \times 10^{-7}$ & $3.18 \times 10^{-6}$ & 9 \\
\hline MCM4 & 2.84 & $1.68 \times 10^{-9}$ & $6.72 \times 10^{-8}$ & 9 \\
\hline ZВТВ16* & -3.85 & $1.84 \times 10^{-8}$ & $5.17 \times 10^{-7}$ & 9 \\
\hline$G R K 5^{*}$ & -3.73 & $9.68 \times 10^{-13}$ & $1.38 \times 10^{-10}$ & 8 \\
\hline$A D R B 2^{*}$ & -4.67 & $3.52 \times 10^{-12}$ & $3.99 \times 10^{-10}$ & 8 \\
\hline$B U B 1 B$ & 3.45 & $4.58 \times 10^{-7}$ & $7.76 \times 10^{-6}$ & 7 \\
\hline$S P T B N 1^{*}$ & -2.26 & $9.16 \times 10^{-13}$ & $1.32 \times 10^{-10}$ & 7 \\
\hline$C C N B 2$ & 2.64 & $1.37 \times 10^{-8}$ & $4.02 \times 10^{-7}$ & 7 \\
\hline RFC4 & 3.41 & $1.81 \times 10^{-10}$ & $1.03 \times 10^{-8}$ & 7 \\
\hline COL $3 A 1$ & 2.56 & $8.70 \times 10^{-9}$ & $2.68 \times 10^{-7}$ & 7 \\
\hline$K A T 2 B$ & -2.19 & $5.49 \times 10^{-5}$ & $3.92 \times 10^{-4}$ & 6 \\
\hline FEN1 & 2.63 & $4.44 \times 10^{-8}$ & $1.09 \times 10^{-6}$ & 6 \\
\hline FHL2* & 2.18 & $1.22 \times 10^{-6}$ & $1.75 \times 10^{-5}$ & 6 \\
\hline$C K S 1 B$ & 2.71 & $7.25 \times 10^{-12}$ & $7.00 \times 10^{-10}$ & 6 \\
\hline$M A D 2 L 1$ & 3.77 & $2.02 \times 10^{-10}$ & $1.13 \times 10^{-8}$ & 6 \\
\hline NDC 80 & 2.63 & $1.18 \times 10^{-6}$ & $1.68 \times 10^{-5}$ & 6 \\
\hline$M M P 9$ & 3.48 & $1.93 \times 10^{-8}$ & $5.40 \times 10^{-7}$ & 6 \\
\hline$H M G A l$ & 2.35 & $1.76 \times 10^{-9}$ & $7.00 \times 10^{-8}$ & 6 \\
\hline SRPK1 & 2.24 & $9.91 \times 10^{-10}$ & $4.33 \times 10^{-8}$ & 6 \\
\hline$T E K^{*}$ & -3.99 & $9.85 \times 10^{-17}$ & $7.29 \times 10^{-14}$ & 5 \\
\hline$G M N N$ & 2.51 & $1.86 \times 10^{-7}$ & $3.63 \times 10^{-6}$ & 5 \\
\hline$N M E 1^{*}$ & 3.56 & $4.41 \times 10^{-11}$ & $3.29 \times 10^{-9}$ & 5 \\
\hline$U B E 2 C$ & 3.10 & $3.06 \times 10^{-9}$ & $1.10 \times 10^{-7}$ & 5 \\
\hline$M R C 1$ & -3.08 & $4.36 \times 10^{-6}$ & $5.04 \times 10^{-5}$ & 5 \\
\hline COL5A1 & 2.62 & $5.15 \times 10^{-7}$ & $8.46 \times 10^{-6}$ & 5 \\
\hline COL1A2 & 2.69 & $1.79 \times 10^{-9}$ & $7.07 \times 10^{-8}$ & 5 \\
\hline$M M P 1$ & 5.25 & $1.51 \times 10^{-6}$ & $2.08 \times 10^{-5}$ & 5 \\
\hline BIRC5 & 2.97 & $1.73 \times 10^{-7}$ & $3.41 \times 10^{-6}$ & 5 \\
\hline$P P A R G$ & -2.07 & $1.14 \times 10^{-6}$ & $1.64 \times 10^{-5}$ & 5 \\
\hline$C D 93^{*}$ & -2.28 & $2.67 \times 10^{-11}$ & $2.09 \times 10^{-9}$ & 4 \\
\hline$F 8^{*}$ & -2.80 & $1.26 \times 10^{-8}$ & $3.71 \times 10^{-7}$ & 4 \\
\hline$N R 4 A 2$ & -2.01 & $1.44 \times 10^{-3}$ & $5.42 \times 10^{-3}$ & 4 \\
\hline THBS2* & 3.49 & $3.63 \times 10^{-10}$ & $1.87 \times 10^{-8}$ & 4 \\
\hline$C D H 5^{*}$ & -3.92 & $3.51 \times 10^{-12}$ & $3.99 \times 10^{-10}$ & 4 \\
\hline COL5A2 & 2.67 & $5.06 \times 10^{-7}$ & $8.34 \times 10^{-6}$ & 4 \\
\hline$B M P 2$ & -2.58 & $9.42 \times 10^{-6}$ & $9.45 \times 10^{-5}$ & 4 \\
\hline$A G T R 1^{*}$ & -4.10 & $4.77 \times 10^{-14}$ & $1.26 \times 10^{-11}$ & 4 \\
\hline PTTG1 & 2.45 & $1.62 \times 10^{-9}$ & $6.55 \times 10^{-8}$ & 4 \\
\hline$M F A P 2$ & 2.72 & $7.23 \times 10^{-6}$ & $7.65 \times 10^{-5}$ & 4 \\
\hline ZWINT & 3.05 & $1.66 \times 10^{-8}$ & $4.76 \times 10^{-7}$ & 4 \\
\hline
\end{tabular}

*Genes also identified in the protein-protein interaction network for never-smoker patients (Table I). 


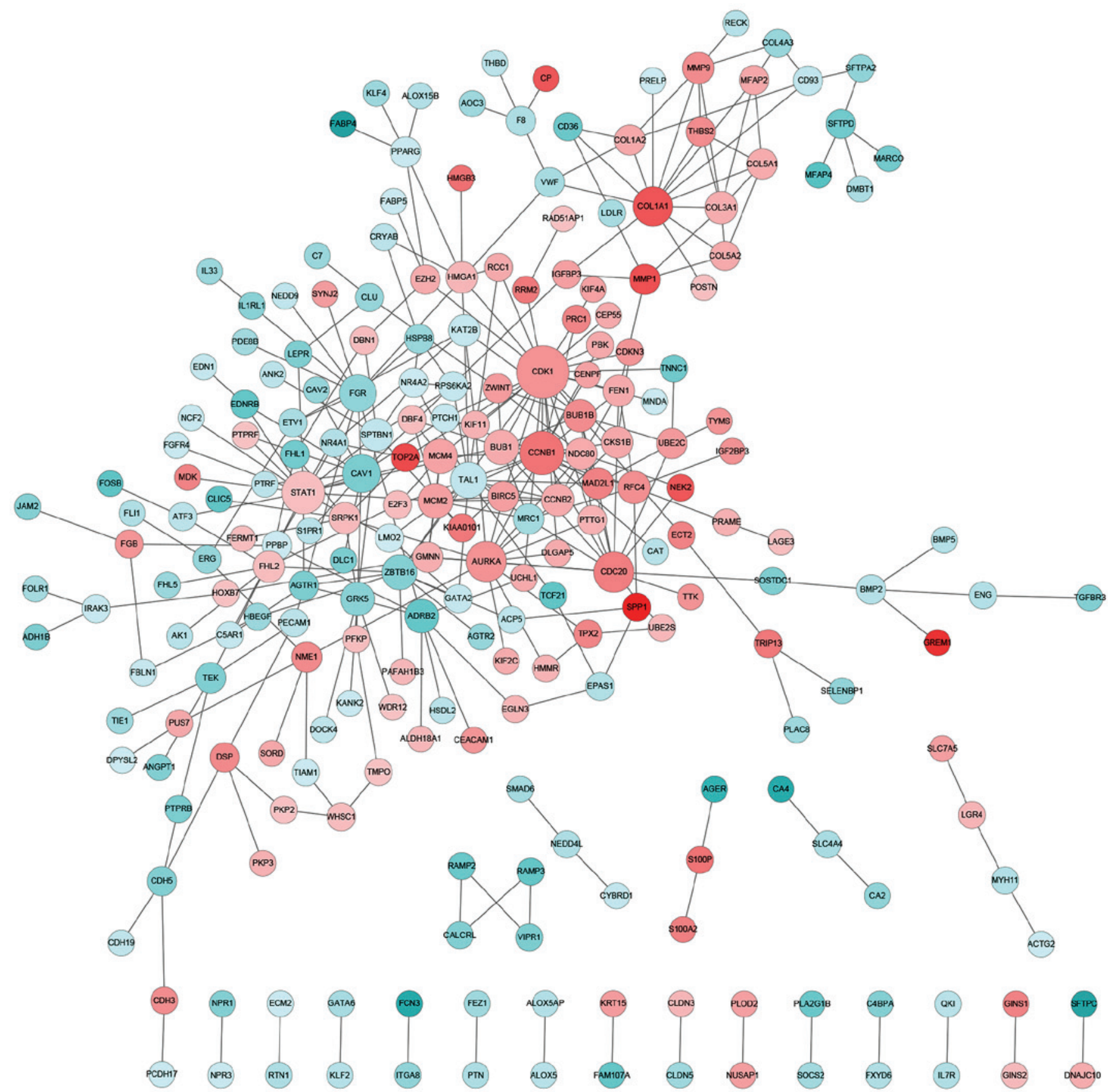

Figure 4. Interaction networks of DEGs involved in lung carcinogenesis in CS patients. Co-expression protein-protein interaction networks that comprise significant DEGs identified for the CS group. Nodes represent DEG-encoded products, with blue indicating downregulation and red indicating upregulation. DEG, differentially-expressed gene; CS, current smoker.

has previously been reported to be to associated with lung cancer (21), was found to be significantly upregulated as a central interactor with 25 other DEGs, indicating an aberrantly enhanced cell cycle transition and progression that is likely to contribute to the pathogenesis of lung adenocarcinoma. Furthermore, distinct lung tumor biopsies and parallel normal tissues from the NS or CS patients were clustered together according to the overall DEG signature (Fig. 5). Among the participants of the identified PPI networks, $F Y N$ and $F L T 1$ were specific to the NS group, whereas CDK1, CCNB1, STAT1, AURKA and CDC20 were unique to the CS patients (Table II and III). However, numerous PPI network-relevant DEGs were shared by the NS and CS groups, including CAVI and FGR (Table II and III). Indeed, previous studies have implicated $C A V I$ as a tumor suppressor that when downregulated, enhances cancer-endothelium interaction and lung cancer progression $(22,23)$. Together, these results indicate that there are smoking-independent and-dependent PPI networks that are mechanistically responsible for lung carcinogenesis.

GO and KEGG analysis of DEGs. Systemic integration of cellular functions of the DEGs into biologically meaningful processes and pathways is greatly informative, and may 
Table IV. GO biological processes and KEGG pathways significantly altered in lung adenocarcinoma compared with normal controls for NS and CS patients.

Altered in adenocarcinoma, $\mathrm{n}$

Item

Threshold P-value

\begin{tabular}{cc}
\hline NS group & CS group \\
676 & 854 \\
17 & 19
\end{tabular}

GO biological processes

$<0.01$

$<0.05$

GO, Gene Ontology; KEGG, Kyoto Encyclopedia of Genes and Genomes; NS, never-smoker; CS, current smoker.

Table V. Significantly altered GO biological processes in tumor samples from never-smoker patients.

\begin{tabular}{llll}
\hline GO-BP-ID & P-value & Count & \multicolumn{1}{c}{ Term } \\
\hline GO:0044707 & $1.66 \times 10^{-17}$ & 224 & Single-multicellular organism process \\
GO:0016477 & $7.43 \times 10^{-16}$ & 70 & Cell migration \\
GO:0008283 & $3.07 \times 10^{-11}$ & 88 & Cell proliferation \\
\hline
\end{tabular}

GO, Gene Ontology; BP, biological process.

Table VI. Significantly altered KEGG pathways in tumor samples from never-smoker patients.

\begin{tabular}{lccc}
\hline KEGG-ID & P-value & Count & Term \\
\hline 4512 & $1.32 \times 10^{-4}$ & 10 & ECM-receptor interaction \\
4510 & $1.61 \times 10^{-4}$ & 16 & Focal adhesion \\
\hline
\end{tabular}

KEGG, Kyoto Encyclopedia of Genes and Genomes; ECM, extracellular matrix.
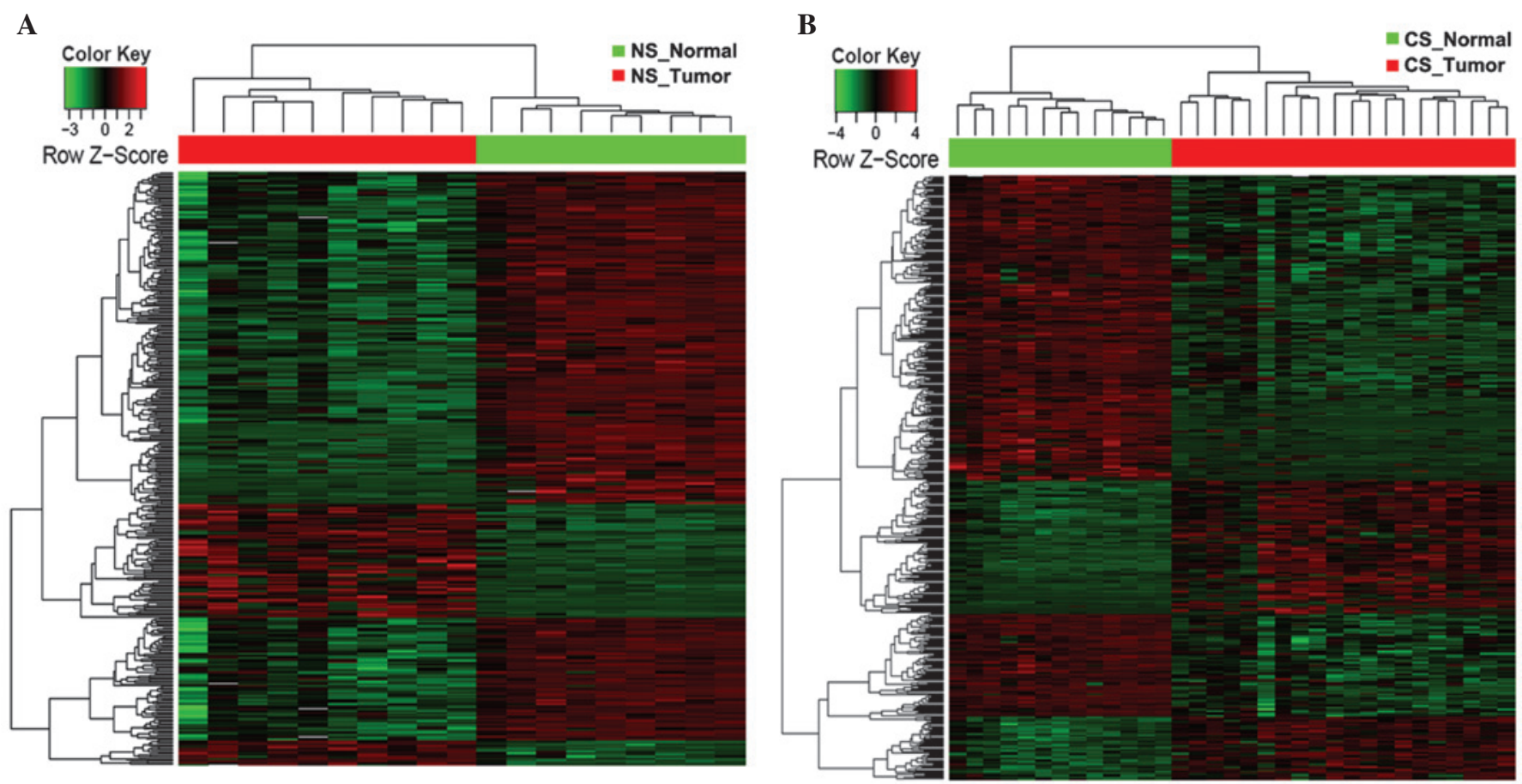

Figure 5. Heatmap and hierarchical clustering based upon expression signatures for the (A) NS and (B) CS groups. Red, relative high expression; green, relative low expression. NS, never-smoker; CS, current smoker. 
A

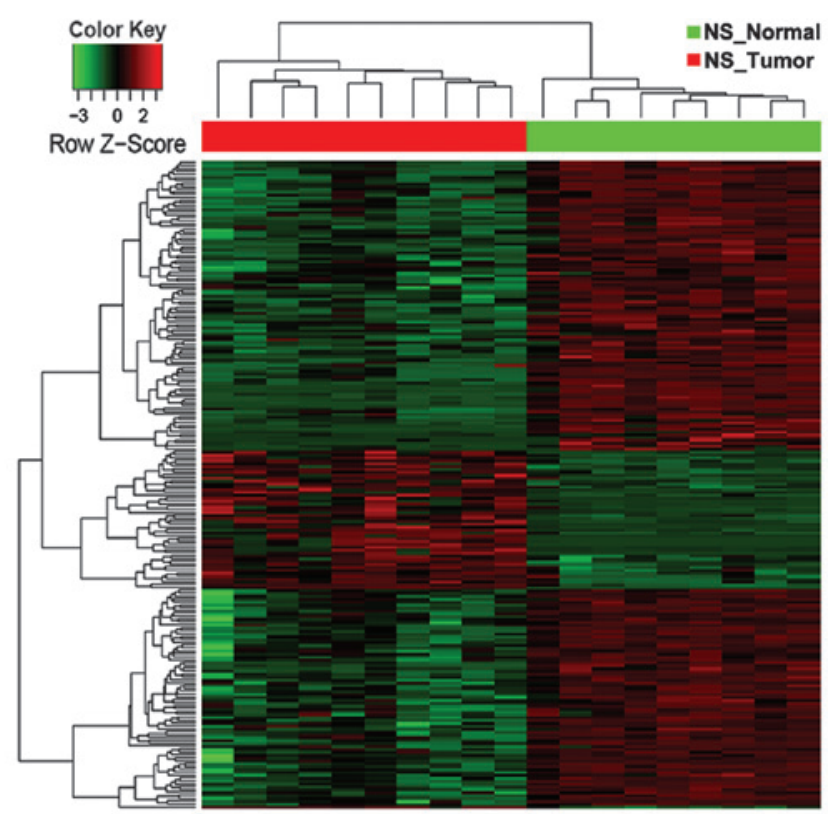

B

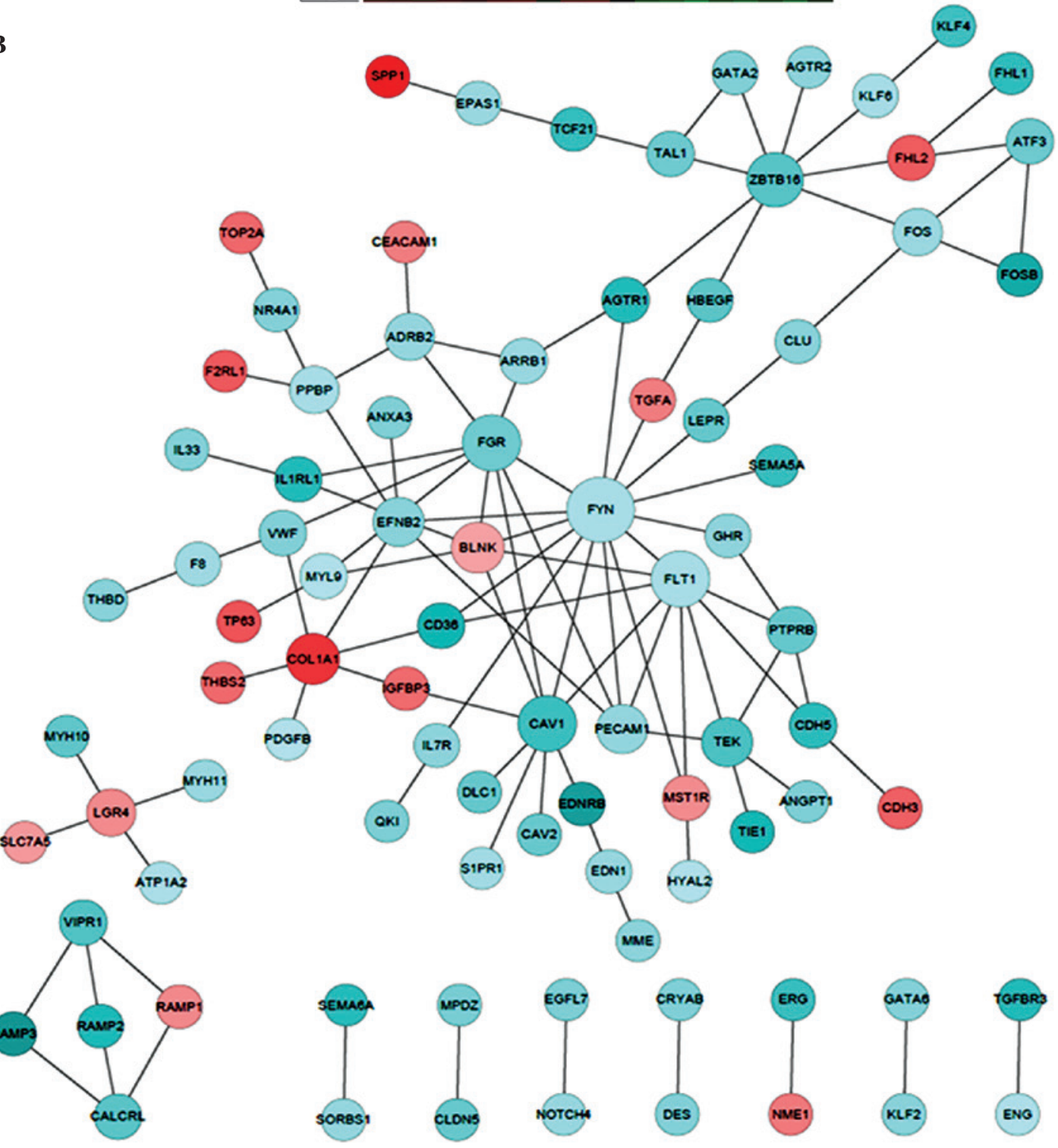

Figure 6. Heatmap and corresponding biological network of DEGs involved in significantly altered Gene Ontology biological processes in lung tumor samples from NS patients. (A) Hierarchical clustering of samples (10 tumor biopsies and 9 normal controls) according to the expression profile involved in single-multicellular organism process (GO:0044707). Red, relative high expression; green, relative low expression. (B) Biological network constructed based on the direct interaction among DEGs for single-multicellular organism process. Each node represents a DEG, with red indicating upregulation and blue indicating downregulation. DEG, differentially-expressed gene; NS, never-smoker. 
A
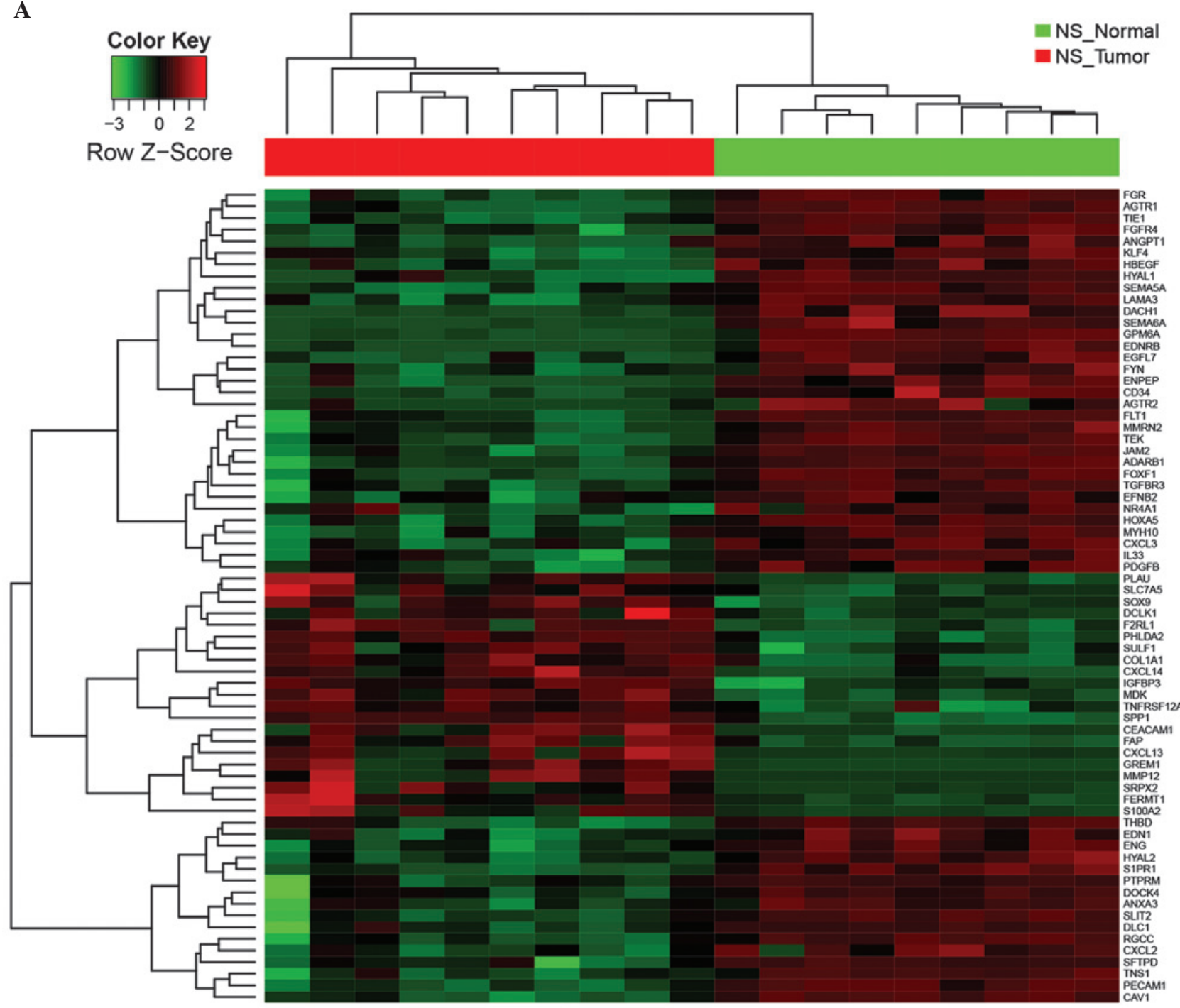

B

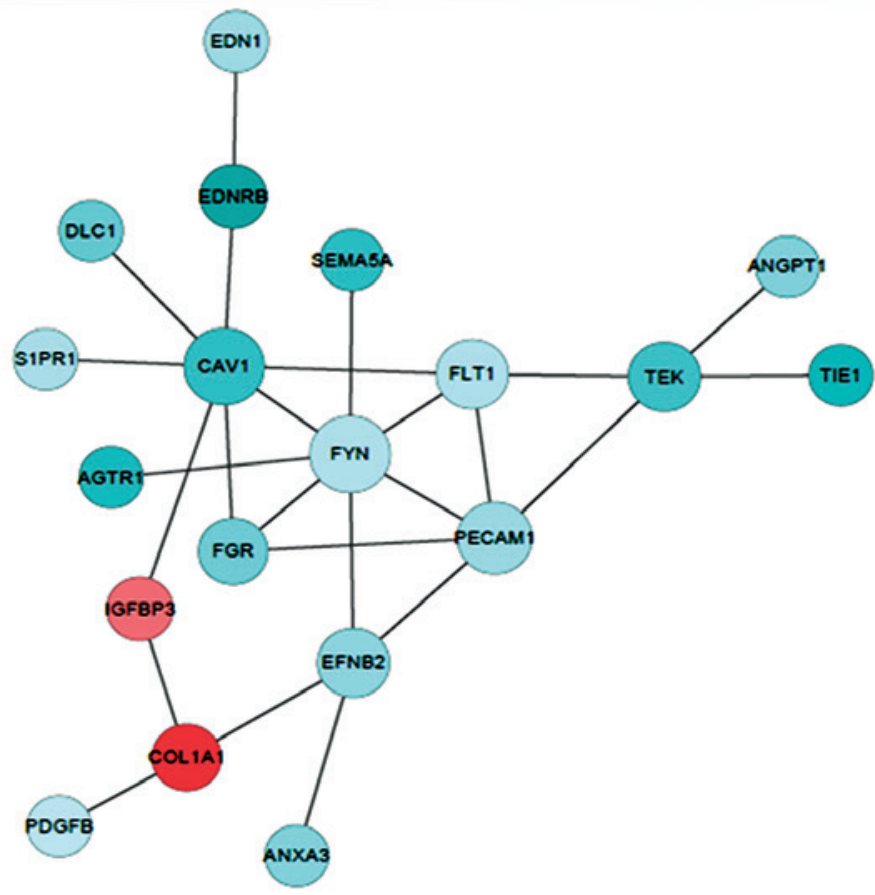

Figure 7. Heatmap and corresponding biological network of DEGs involved in significantly altered Gene Ontology biological processes in lung tumor samples from NS patients. (A) Hierarchical clustering of samples (10 tumor biopsies and 9 normal controls) according to the expression profile involved in cell migration (GO:0016477). Red, relative high expression; green, relative low expression. (B) The biological network was constructed based on the direct interaction among DEGs for cell migration. Each node represents a DEG, with red indicating upregulation and blue indicating downregulation. DEG, differentially-expressed gene; NS, never-smoker. 
A

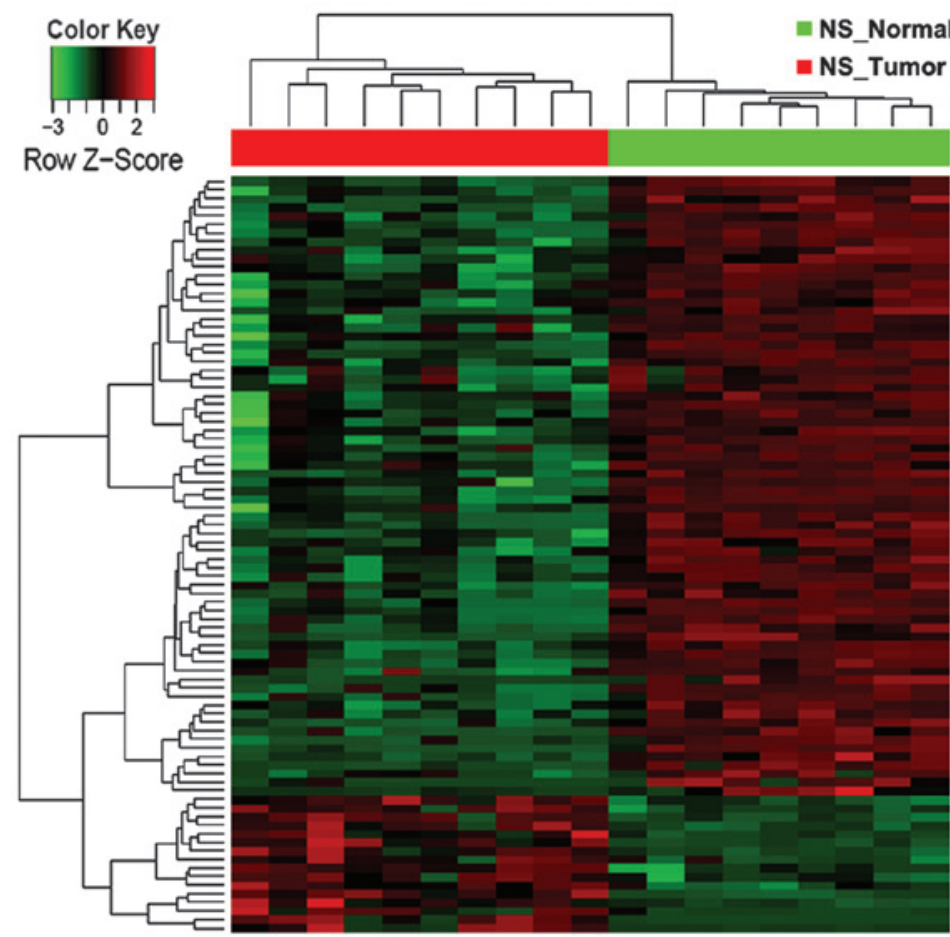

B

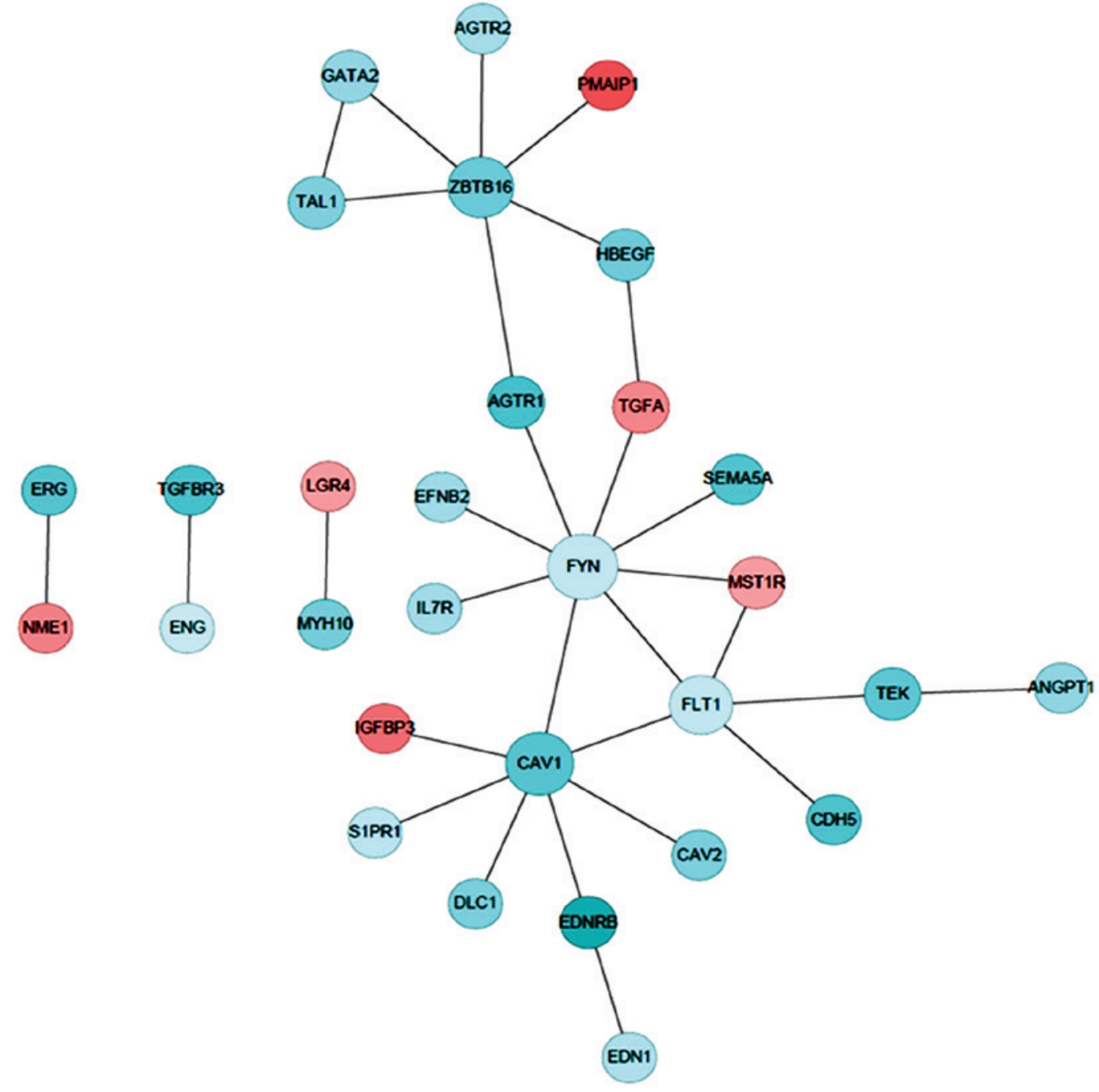

Figure 8. Heatmap and corresponding biological network of DEGs involved in significantly altered Gene Ontology biological processes in lung tumor samples from NS patients. (A) Hierarchical clustering of samples (10 tumor biopsies and 9 normal controls) according to the expression profile involved in cell proliferation (GO:0008283). Red, relative high expression; green, relative low expression. (B) The biological network was constructed based on the direct interaction among DEGs for cell proliferation. Each node represents a DEG, with red indicating upregulation and blue indicating downregulation. DEG, differentially-expressed gene; NS, never-smoker. 


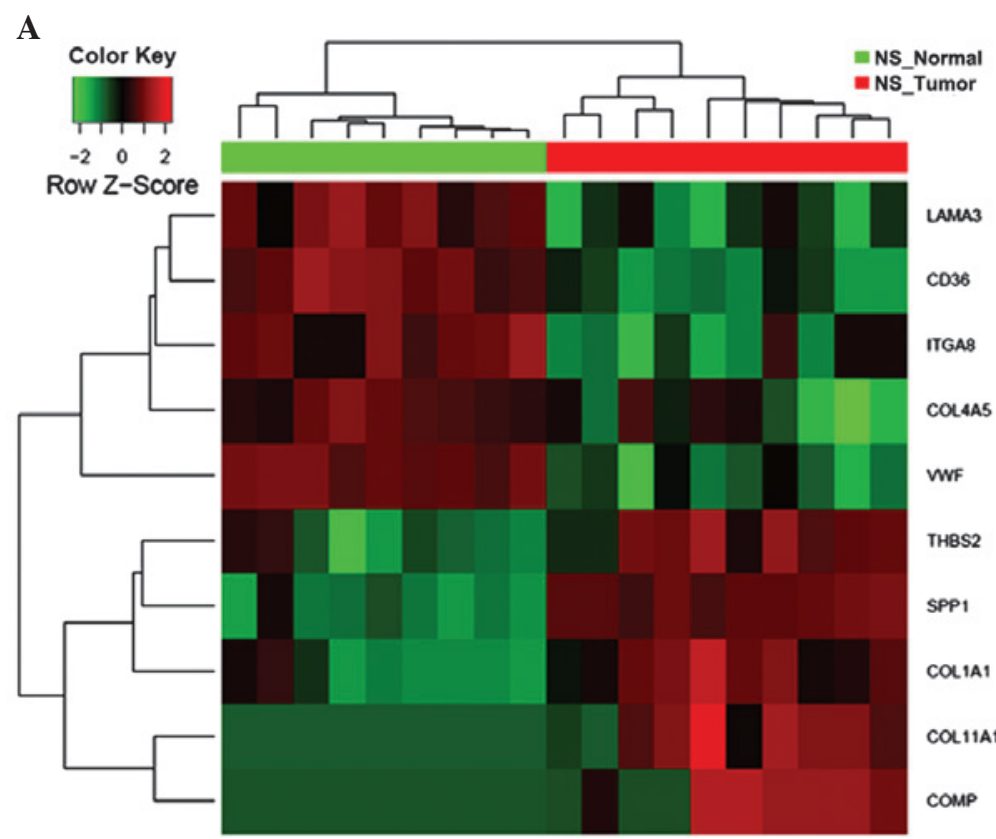

C
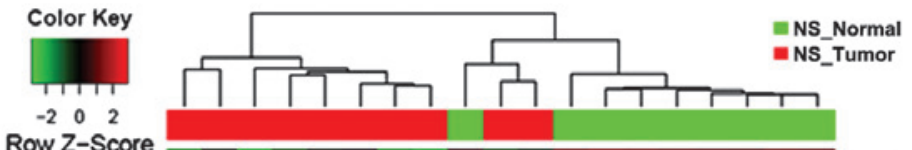

(-

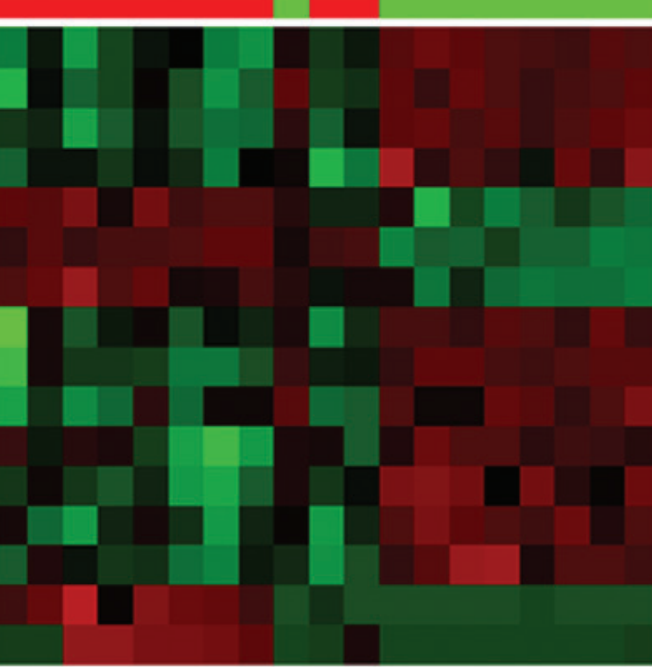

CAV2
WWF
CAV1
MYI
THBS2
SPP1
COL1A1
AKT3
FLT1
ITGA8
COLAS
POGFB
LAMA3
FYN
COL11A1
COMP

B

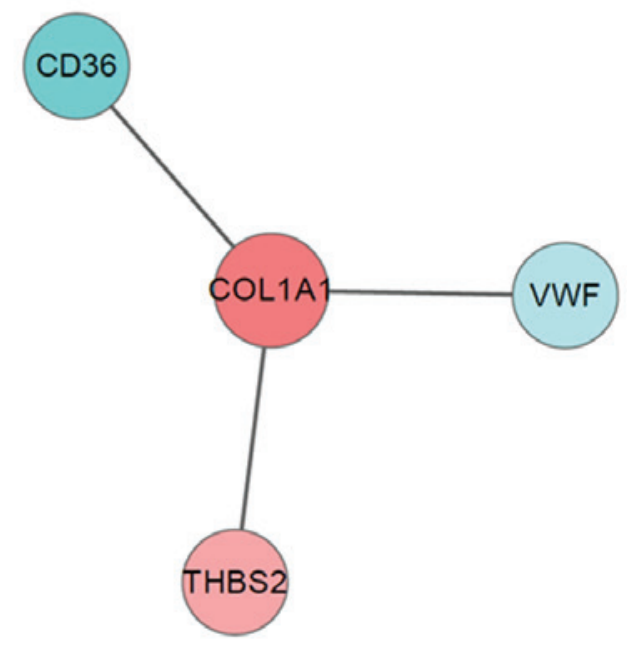

D
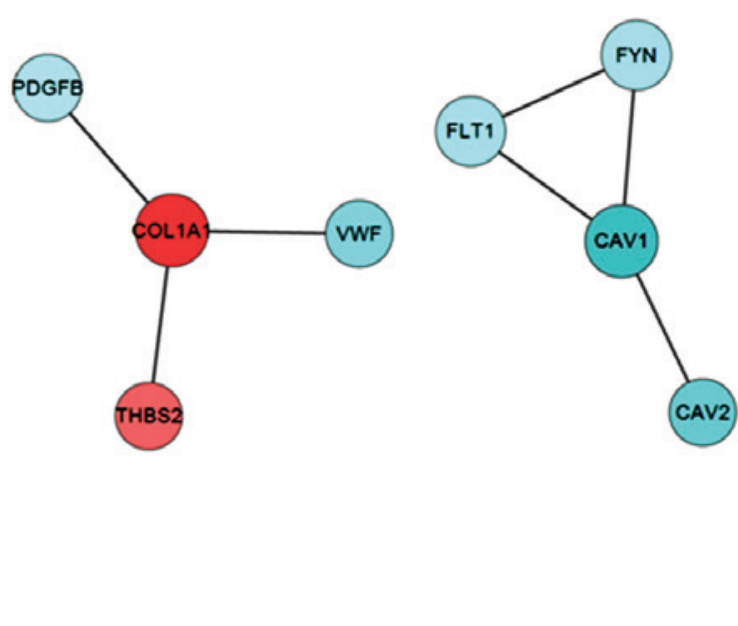

Figure 9. Heatmap and biological network of DEGs involved in significantly altered KEGG pathways in lung tumor samples from NS patients. All samples (10 tumor biopsies and 9 normal controls) were hierarchically clustered according to the expression profile involved in (A) ECM-receptor interaction (KEGG:04512) and (C) focal adhesion (KEGG:04510). Red, relative high expression; green, relative low expression. The biological network was constructed based on the direct interaction among DEGs for (B) ECM-receptor interaction and (D) focal adhesion. Each node represents a DEG, with red indicating upregulation and blue indicating downregulation. DEG, differentially-expressed gene; KEGG, Kyoto Encyclopedia of Genes and Genomes; NS, never-smoker; ECM, extracellular matrix.

reveal underlying mechanistic and therapeutic targets in lung carcinogenesis. Given the list of DEGs identified from tumor samples compared with normal tissues in NS and CS patients, over-representation analysis was performed to uncover enriched terms based on curated GO and KEGG vocabularies, which are two similar biological function-focused databases with distinct infrastructures $(24,25)$. In the lung tumor biopsies from NS patients, a total of 676 GO biological processes and 17 KEGG pathways were revealed to be significantly affected (Table IV). The top three GO terms were 'single-multicellular organism process', ‘cell migration' and 'cell proliferation' (Table V), whilst the most significantly enriched KEGG pathways included 'extracellular matrix (ECM)-receptor interaction' and 'focal adhesion' (Table VI). Interaction between $F Y N$, $C A V 1$ and FLT1 constitutes the central hub of these relevant PPI networks, further indicating their potential contributing roles in the early stages of lung carcinogenesis. In addition, the expression patterns of DEGs that constitute these significantly altered GO and KEGG terms differed markedly 
A
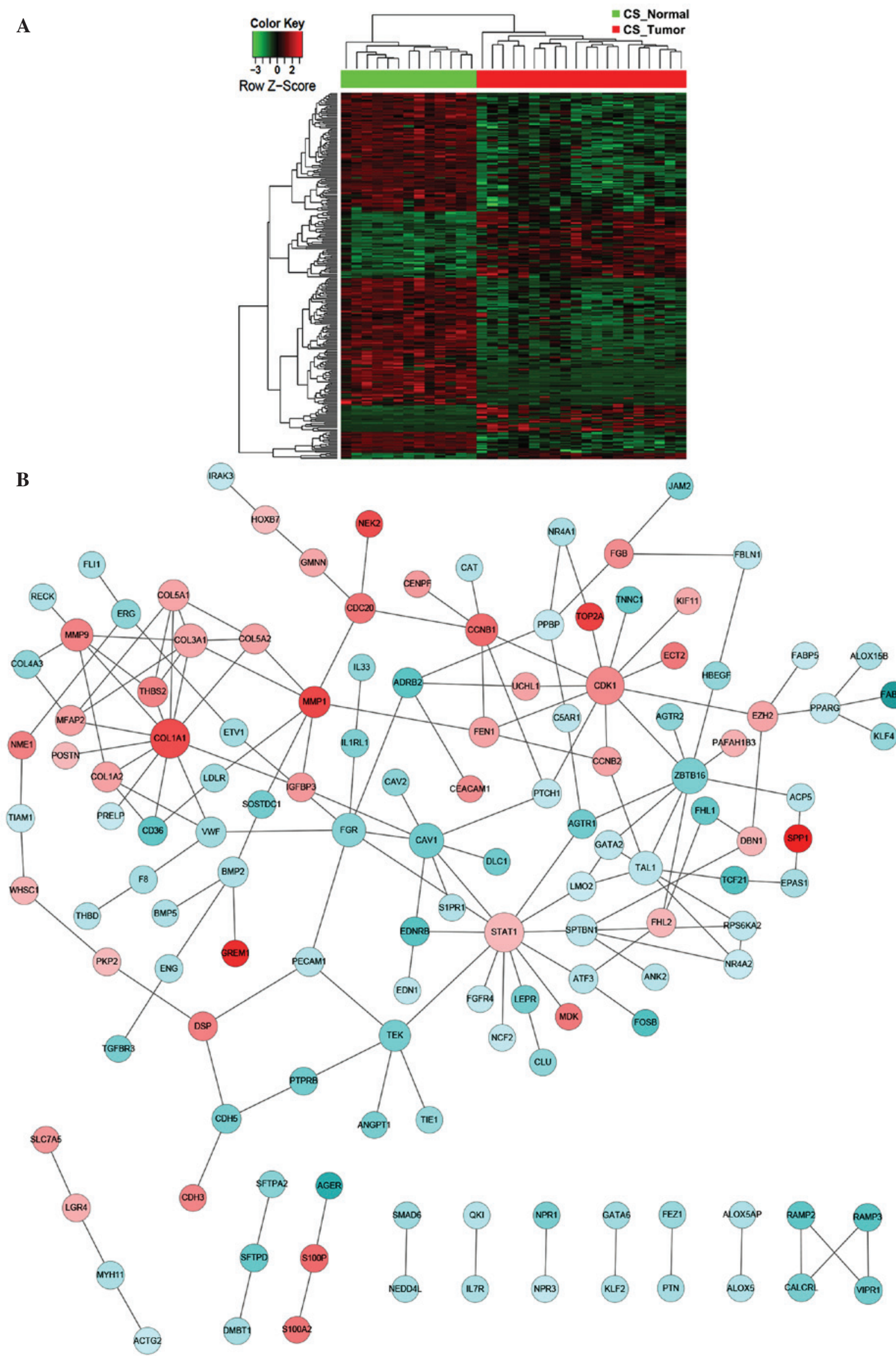

Figure 10. Heatmap and corresponding biological network of DEGs involved in significantly altered Gene Ontology biological processes in lung tumor samples from CS patients. (A) Hierarchical clustering of samples (10 tumor biopsies and 9 normal controls) according to the expression profile involved in single-multicellular organism process (GO:0044707). Red, relative high expression; green, relative low expression. (B) The biological network was constructed based on the direct interaction among DEGs for single-multicellular organism process. Each node represents a DEG, with red indicating upregulation and blue indicating downregulation. DEG, differentially-expressed gene; CS, current smoker. 
A

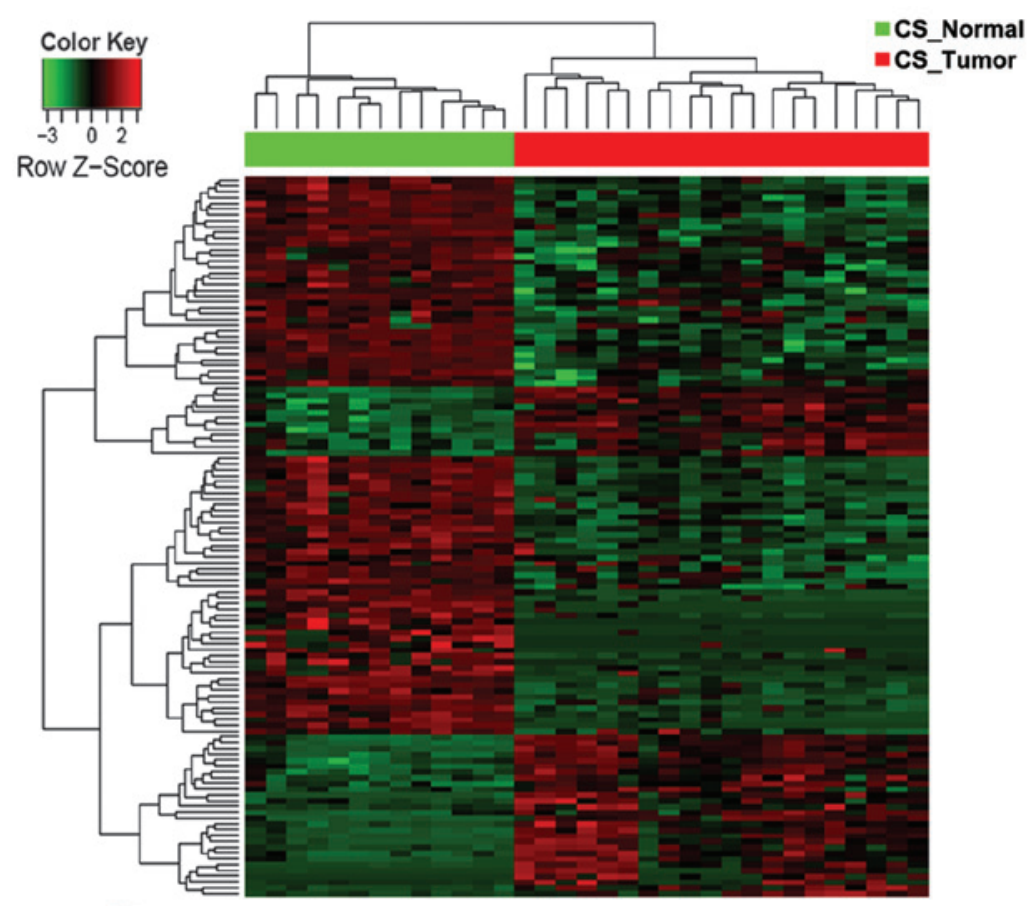

B
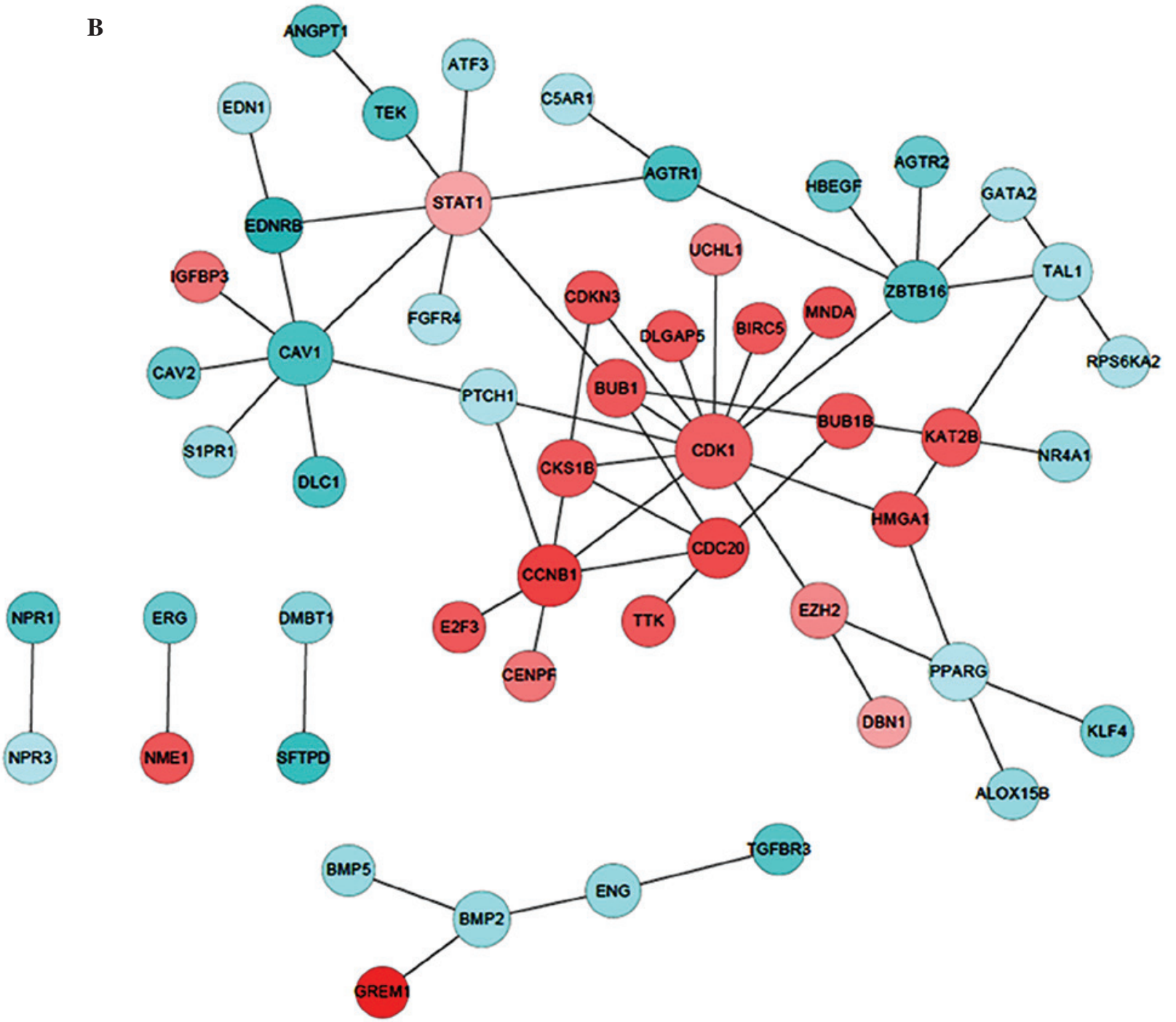

Figure 11. Heatmap and corresponding biological network of DEGs involved in significantly altered Gene Ontology biological processes in lung tumor samples from CS patients. (A) Hierarchical clustering of samples (10 tumor biopsies and 9 normal controls) according to the expression profile involved in cell proliferation (GO:0008283). Red, relative high expression; green, relative low expression. (B) The biological network was constructed based on the direct interaction among DEGs for cell proliferation. Each node represents a DEG, with red indicating upregulation and blue indicating downregulation. DEG, differentially-expressed gene; CS, current smoker. 
A

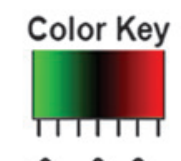

$\begin{array}{lll}-3 & 0 & 2\end{array}$

Row Z-Score

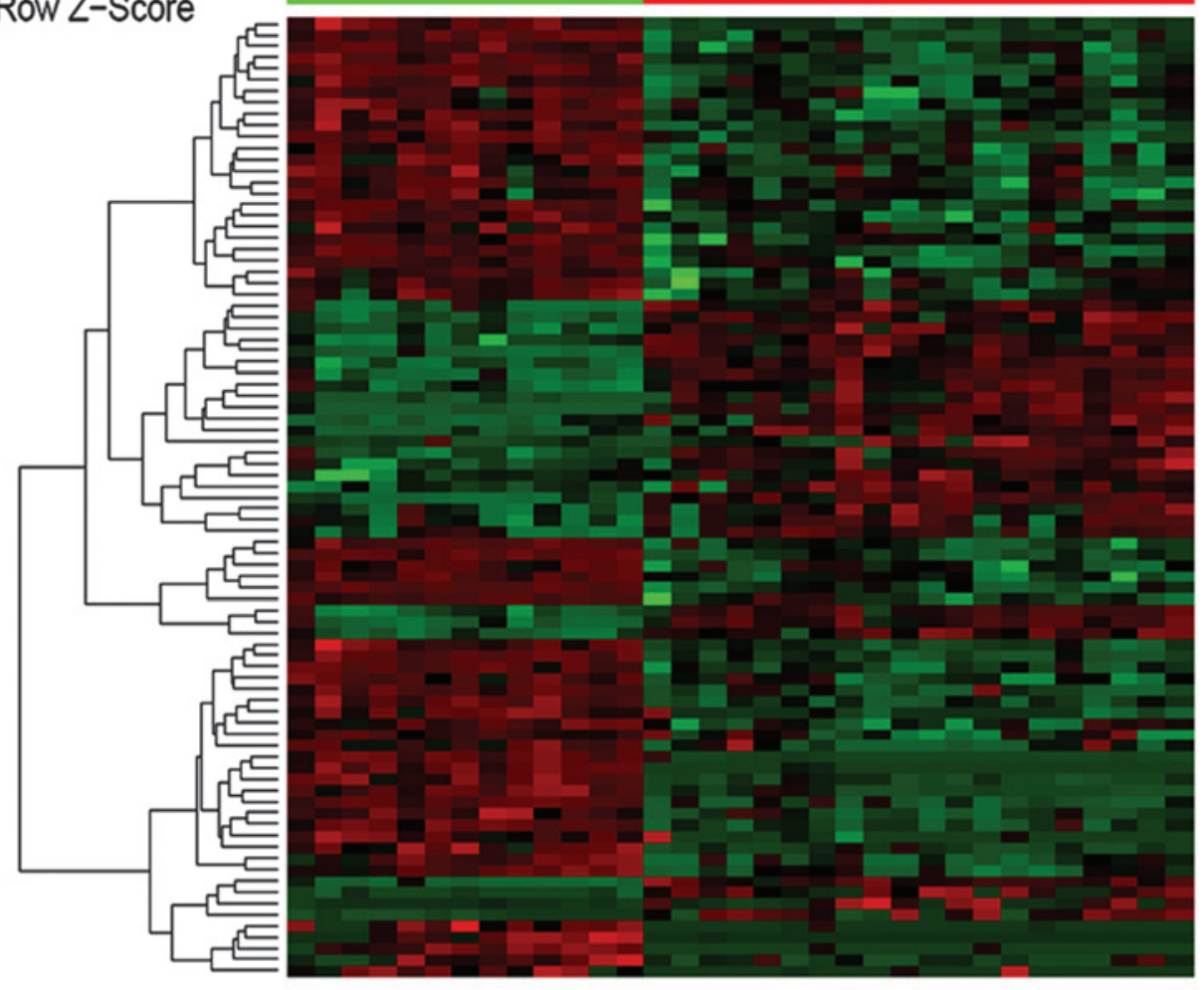

B

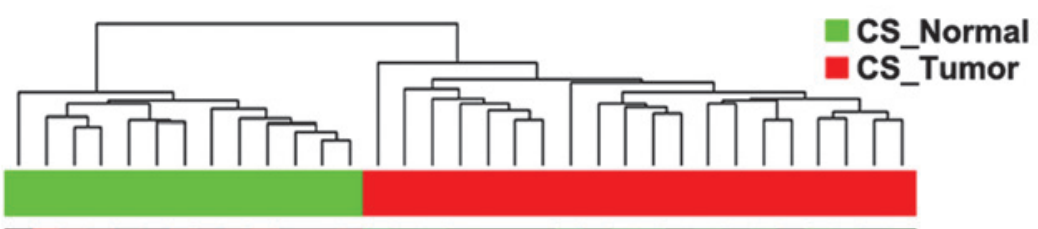

nCS_Tumor

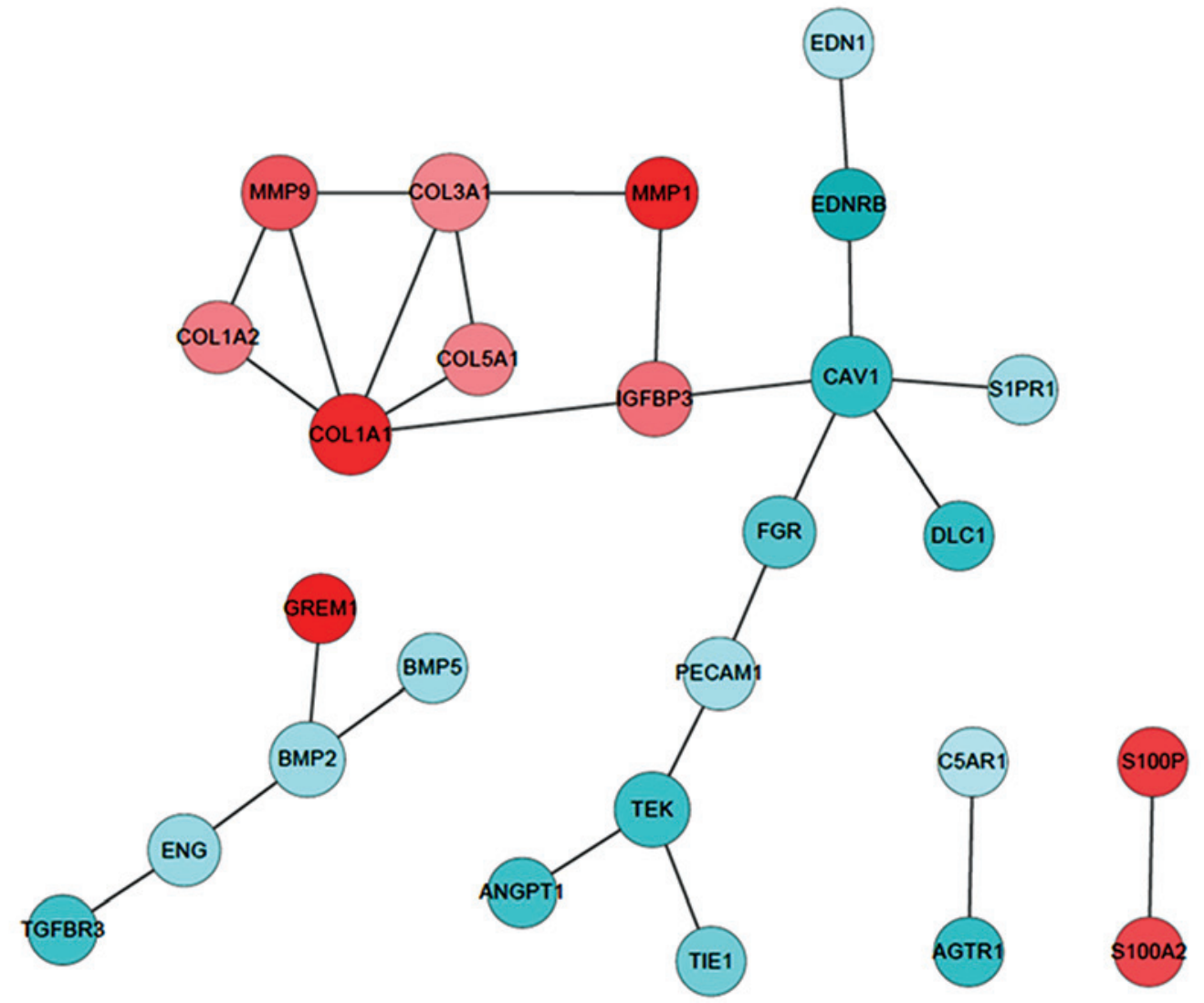

Figure 12. Heatmap and corresponding biological network of DEGs involved in significantly altered Gene Ontology biological processes in lung tumor samples from CS patients. (A) Hierarchical clustering of samples (10 tumor biopsies and 9 normal controls) according to the expression profile involved in cell migration (GO:0016477). Red, relative high expression; green, relative low expression. (B) The biological network was constructed based on the direct interaction among DEGs for cell migration. Each node represents a DEG, with red indicating upregulation and blue indicating downregulation. DEG, differentially-expressed gene; CS, current smoker. 
A

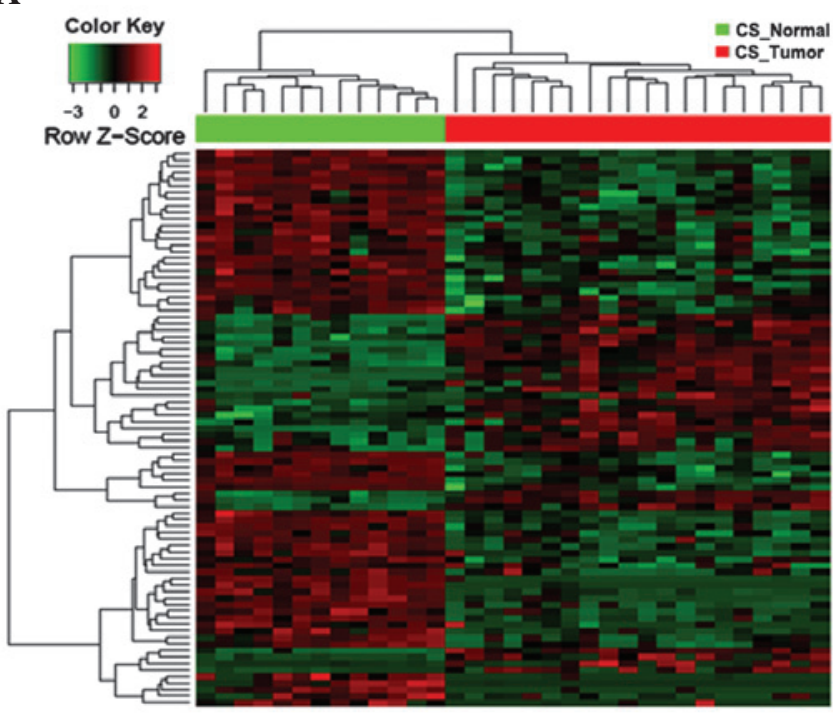

B

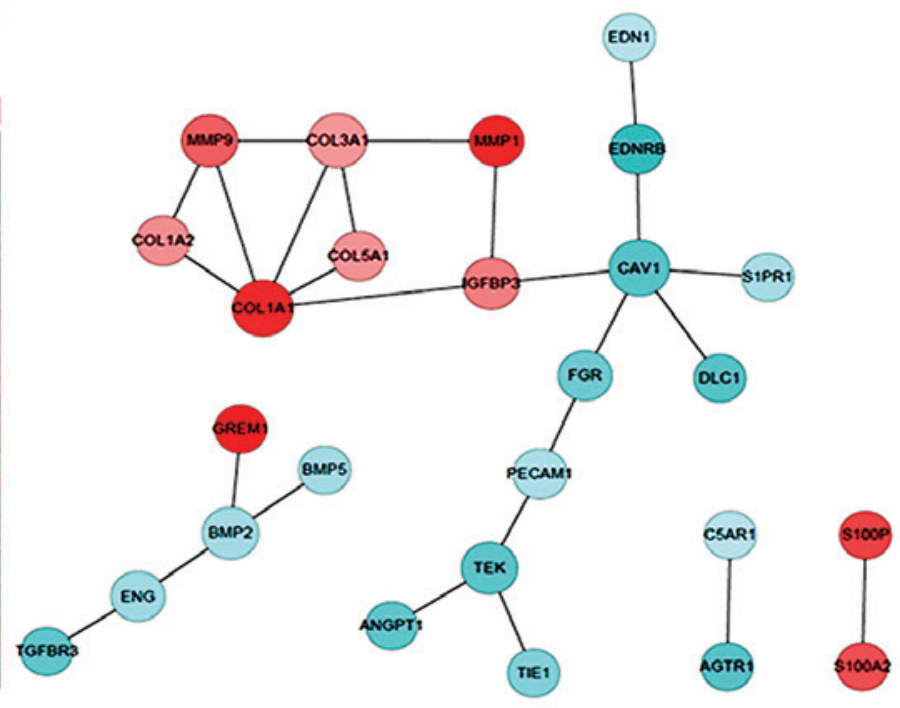

D
C

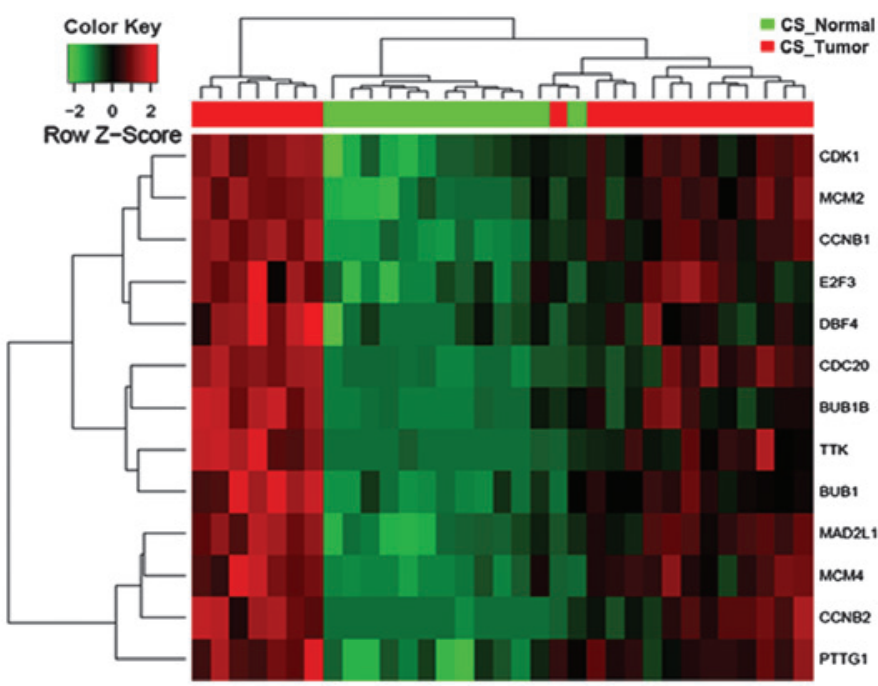

\section{.}

CNB1

20

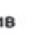

81
$\mathbf{E}$

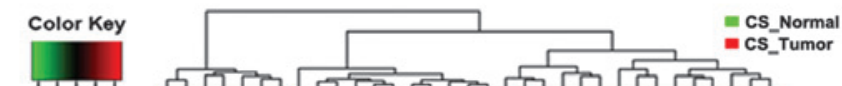
Row Z-Score

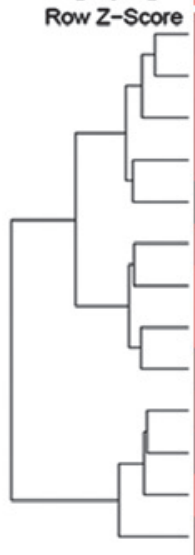

\section{_Tormal}

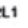

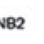

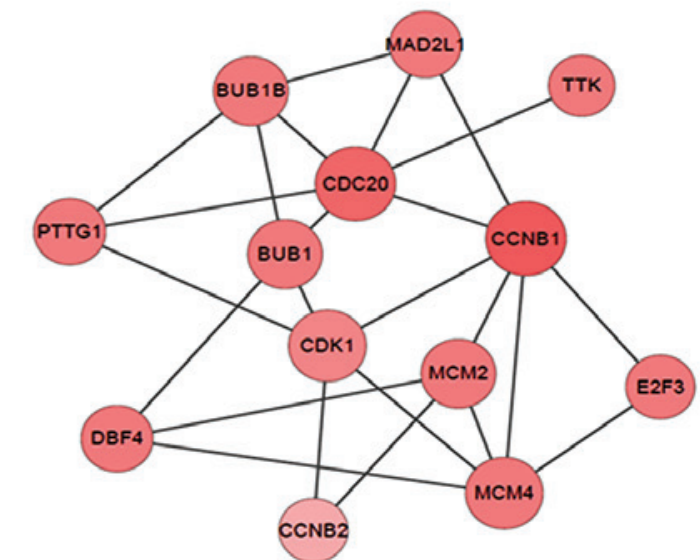

$\mathbf{F}$

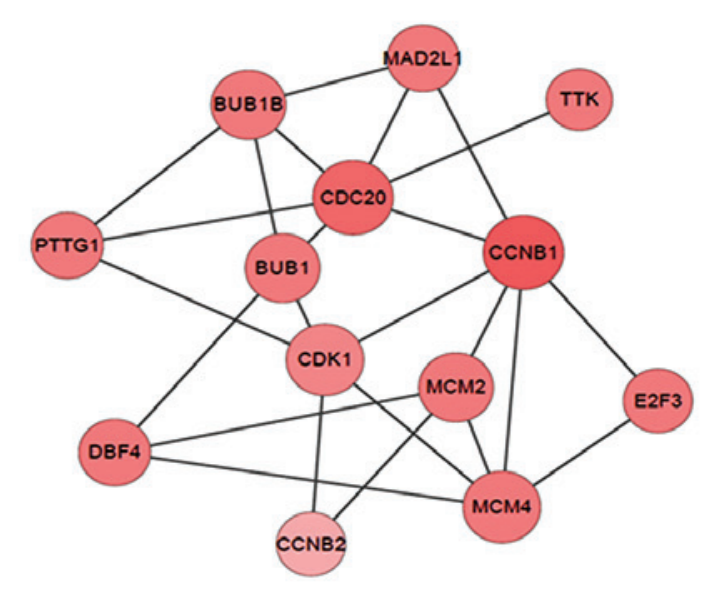

Figure 13. Heatmap and biological network of DEGs involved in significantly altered KEGG pathways in lung tumor samples from CS patients. All samples (13 tumor biopsies and 20 normal controls) were hierarchically clustered according to the expression profile involved in (A) ECM-receptor interaction (KEGG:04512), (C) cell cycle (KEGG: 04110) and (E) p53 signaling pathway (KEGG: 04115). Red, relative high expression; green, relative low expression. The biological network was constructed based on the direct interaction among DEGs for (B) ECM-receptor interaction, (D) cell cycle and (F) p53 signaling pathway. Each node represents a DEG, with red indicating upregulation and blue indicating downregulation. DEG, differentially-expressed gene; KEGG, Kyoto Encyclopedia of Genes and Genomes; CS, current smoker; ECM, extracellular matrix. 
Table VII. Significantly altered GO biological processes in tumor samples from current smoker patients.

\begin{tabular}{llll}
\hline GO-BP-ID & P-value & Count & \multicolumn{1}{c}{ Term } \\
\hline GO:0044707 & $5.90 \times 10^{-23}$ & 292 & Single-multicellular organism process \\
GO:0008283 & $2.37 \times 10^{-18}$ & 124 & Cell proliferation \\
GO:0016477 & $3.29 \times 10^{-17}$ & 85 & Cell migration \\
\hline
\end{tabular}

GO, Gene Ontology; BP, biological process.

Table VIII. Significantly altered KEGG pathways in tumor samples from current smoker patients.

\begin{tabular}{llcl}
\hline KEGG-ID & P-value & Count & Term \\
\hline 4512 & $1.58 \times 10^{-5}$ & 13 & ECM-receptor interaction \\
4110 & $7.88 \times 10^{-4}$ & 13 & Cell cycle \\
4115 & $4.35 \times 10^{-2}$ & 6 & p53 signaling pathway \\
\hline
\end{tabular}

KEGG, Kyoto Encyclopedia of Genes and Genomes; ECM, extracellular matrix.

between the tumor tissues and normal controls from the NS patients, as demonstrated by the hierarchical clustering of samples in the heat maps (Figs. 6-9).

For lung tumor samples from CS patients, a total of 854 GO biological processes and 19 KEGG pathways were identified to be relevant (Table IV). Among these, 'single-multicellular organism process', 'cell proliferation' and 'cell migration' were identified to be over-represented GO terms, and 'ECM-receptor interaction' an over-represented KEGG pathway (Table VII and VIII). By contrast, 'cell cycle' and 'p53 signaling pathways' were exclusively enriched in CS patients (Table VIII). The expression profile of DEGs involved in these biological processes and pathways may also be utilized to distinguish lung tumor samples from normal controls, as shown by the constructed heat maps (Figs. 10-13). In contrast to that in the NS patients, the core interaction network of the enriched terms for CS patients was established on two central players: CDK1 and COL1Al (Figs. 10-13), which dictate cell cycle control and collagen metabolism, respectively. Taken together, these results indicate that the cellular machinery of single-multicellular organism processes, cell proliferation, cell migration and ECM-receptor interaction may contribute to smoking-independent early stages of lung adenocarcinoma, whereas dysregulated cell cycle control and p53 signaling cascades are associated with smoking-induced lung carcinogenesis.

\section{Discussion}

The systemic molecular expression signature induced by smoking in lung cancer patients remains incompletely characterized. In the present study, publicly available microarray expression datasets derived from NS and CS patients with stage I or II lung adenocarcinoma were utilized. Despite a number of previous studies that have molecularly characterized genetic profiles in lung cancer patients with or without smoking history (26-30), the present investigation focused on a relatively larger cohort that comprised 107 tumor samples from 74 patients, thereby providing a more powerful analysis. In contrast to a previous study, in which this dataset was analyzed by comparing gene expression profiles between different individuals (that is, tumor samples from smokers versus non-smokers) (12), the approach adopted in the current study aimed to address questions regarding the smoking-dependent and -independent molecular mechanisms involved in lung carcinogenesis by systemically comparing tumors and adjacent normal tissues within the same individuals, which is likely to produce a better signal-to-noise readout. In addition, although the dataset has previously been analyzed almost exclusively at the gene level (12), the present approach was designed to uncover smoking-induced gene expression patterns and also, more importantly, reveal significantly altered gene sets and biological pathways. Specifically, GC-RMA was utilized, followed by the construction of gene networks to allow the identification of potential targets that may be translatable to therapeutic benefits in the clinical setting. This may explain why novel targets and altered pathways were identified in the current study that were largely distinct from those of the previous study (12).

In the present study, among the genes queried on the platform of the Affymetrix Human Genome U133A microarray, a total of 422 and 534 DEGs were identified in NS and CS patients, respectively, for which significant alteration between tumor biopsies and normal tissues was identified. Notably, the NS and CS groups shared 277 common DEGs, indicating similar pathogenic mechanisms that contribute to lung carcinogenesis independently of smoking status. To confirm the differential expression of DEGs, RT-qPCR was conducted using samples from tumor biopsies and matching healthy controls that were independently collected from lung cancer patients with or without smoking history. The results were highly consistent with the DEG analysis, supporting the utility and validity of this analytical approach. The results 
also revealed that multiple biological processes and pathways, including single-multicellular organism process, cell migration, cell proliferation and ECM-receptor interaction, were significantly affected in lung tumor tissues from the NS and CS patients. However, smoking specifically induced altered expression of 257 DEGs that were not identified in the NS patients, suggesting unique downstream molecular and genetic networks that are associated with smoking. Consistently, cell cycle and p53 signaling pathways were significantly altered in lung tumor samples from CS and not NS patients.

One of the most noteworthy DEGs identified in NS and CS patients is caveolin-1 (CAVI), which encodes a scaffolding component of the caveolae plasma membranes. Multiple independent studies have indicated that $C A V l$ has a tumor-suppressor role in lung cancer and other types of malignancy $(22,23,31-33)$, which is consistent with the current findings, which revealed that the expression of $C A V 1$ was significantly attenuated in lung cancer patients compared with healthy individuals. With regard to the mechanism, studies have demonstrated that CAVI suppresses vascular cell adhesion protein 1-mediated adhesion between human lung cancer cells and endothelial cells by attenuating the production of hydrogen peroxide and hydroxyl radicals (32). In addition, $C A V 1$ interacts with the StAR-related lipid transfer domain of deleted in liver cancer 1 , another tumor suppressor that is frequently mutated in non-small cell lung cancer patients, and forms a complex to inhibit cancer cell migration and neoplastic development (31). However, despite these studies revealing a tumor-reducing role for $C A V 1$, other investigations have revealed that $C A V I$ also possesses a contradictory tumor-promoting function (34-36). In particular, Pancotti et al demonstrated that siRNA-induced $C A V 1$-knockdown resulted in cell cycle arrest in vitro in cell lines derived from metastatic lesions of lung adenocarcinoma and small cell lung carcinoma, and that this was associated with the reduced expression of cyclin DI and CDK4 and the attenuated phosphorylation of Akt (34). Taken together, these studies indicate a multifaceted tumor-regulatory role for $C A V 1$ that is likely to be context-dependent; future investigations are required prior to $C A V l$ being targeted for therapeutic purposes to treat lung cancer and other malignancies.

The present analysis also identified a unique pattern of gene expression exclusively in lung cancer patients with smoking history, including $C D K 1, C C N B 1$, STAT1, $A U R K A$ and $C D C 20$. Notably, all of these upregulated DEGs encode essential regulators that dictate cell cycle control and progression, indicating that smoking induces cell hyperproliferation that contributes to the pathogenesis of lung adenocarcinoma. Indeed, numerous previous studies have established a strong association between cigarette smoking and cell proliferation in various types of malignancies, including lung cancer (37-42). The mitogenic effect is largely mediated by nicotine and its derivatives (the major components of cigarettes) through multiple distinct molecular mechanisms (39). For example, it has been demonstrated that smoking induces production of oxygen radicals that cause generation of cleaved transmembrane amphiregulin, which is subsequently detected by EGFR, thereby resulting in aberrant proliferation of lung epithelial cells (37). In addition, by engaging nicotinic acetylcholine receptors (nAChRs), nicotine exerts a pleiotropic cellular function that includes secretion of growth factors (such as VEGF and platelet-derived growth factor) (43) and initiation of mitogen-activated protein kinase signaling cascades (44). In particular, in non-small cell lung cancers, activation of nAChRs induces recruitment of $\beta$-arrestin to the receptor, which further activates Src and enhances binding of the transcription activators E2F1 and Raf-1 to proliferative promoters (41). Consequently, exposure to nicotine in cigarettes induces abnormal mitogenesis through these various mechanisms that contribute synergistically to the initiation and development of lung adenocarcinoma.

Additional classes of carcinogens from tobacco smoking have also been demonstrated to affect lung tumorigenesis, including polycyclic aromatic hydrocarbons, tobacco-specific nitrosamines and aldehydes. Their potent carcinogenic capability is primarily mediated by imposing genotoxic stress and damage in host cells. For example, formation of DNA adducts is frequently induced, which results in loss-of-function mutation in tumor suppressor genes, such as $p 53(39,45-48)$. p53 is a well-established master regulator for the DNA repair response, cell cycle checkpoint and apoptosis, and numerous studies have implicated a long-standing association between the high incidence of its perturbed expression or function and the development of various types of cancer $(49,50)$. Indeed, the mutational frequency of $p 53$ is considerably higher in smokers ( $55 \%)$ compared with non-smokers $(\sim 25 \%)$ in lung cancer patients, predominantly involving G-to-T transitions $(46,47)$. Consistently, the present study identified the p53 signaling pathway as significantly affected only in CS patients, further supporting a contributing role for smoking in the molecular initiation and progression of lung cancers.

In summary, the present study systemically investigated the molecular alterations that are associated with the pathogenesis of lung adenocarcinoma patients with or without smoking history. Numerous common gene signatures and biological pathways were identified as smoking-independent mechanisms, including the $C A V 1$ gene and pathways that are related to cell migration and proliferation. By contrast, cigarette smoking induces a characteristic gene expression profile involved in cell cycle control and p53 response cascades. Overall, this analysis provided additional molecular knowledge that furthers our understanding of lung tumorigenesis, which may provide potential targets for the therapeutic design of efficacious treatment for lung cancers and other malignancies.

\section{References}

1. Ferlay J, Shin HR, Bray F, Forman D, Mathers C and Parkin DM: Estimates of worldwide burden of cancer in 2008: GLOBOCAN 2008. Int J Cancer 127: 2893-2917, 2010.

2. Surveillance, Epidemiology, and End Results Program: SEER Stat Fact Sheets: Lung and Bronchus Cancer. National Cancer Institute at the National Institutes of Health. http://seer.cancer. gov/statfacts/html/lungb.html. Accessed March 24, 2015.

3. Peto R, Darby S, Deo H, Silcocks P, Whitley E and Doll R: Smoking, smoking cessation, and lung cancer in the UK since 1950: Combination of national statistics with two case-control studies. BMJ 321: 323-329, 2000. 
4. Amos CI, Wu X, Broderick P, Gorlov IP, Gu J, Eisen T, Dong Q, Zhang Q, Gu X, Vijayakrishnan J, et al: Genome-wide association scan of tag SNPs identifies a susceptibility locus for lung cancer at 15q25.1. Nat Genet 40: 616-622, 2008.

5. Cornfield J, Haenszel W, Hammond EC, et al: Smoking and lung cancer: Recent evidence and a discussion of some questions. 1959. Int J Epidemiol 38: 1175-1191, 2009.

6. Shigematsu H, Takahashi T, Nomura M, Majmudar K, Suzuki M, Lee H, Wistuba II, Fong KM, Toyooka S, Shimizu N, et al Somatic mutations of the HER2 kinase domain in lung adenocarcinomas. Cancer Res 65: 1642-1646, 2005.

7. Hecht SS: Tobacco smoke carcinogens and lung cancer. J Natl Cancer Inst 91: 1194-1210, 1999.

8. Imielinski M, Berger AH, Hammerman PS, Hernandez B, Pugh TJ, Hodis E, Cho J, Suh J, Capelletti M, Sivachenko A, et al: Mapping the hallmarks of lung adenocarcinoma with massively parallel sequencing. Cell 150: 1107-1120, 2012.

9. Govindan R, Ding L, Griffith M, et al: Genomic landscape of non-small cell lung cancer in smokers and never-smokers. Cell 150: 1121-1134, 2012

10. Le Calvez F, Mukeria A, Hunt JD, Kelm O, Hung RJ, Tanière $P$, Brennan P, Boffetta P, Zaridze DG and Hainaut P: TP53 and KRAS mutation load and types in lung cancers in relation to tobacco smoke: distinct patterns in never, former, and current smokers. Cancer Res 65: 5076-5083, 2005.

11. Pao W, Miller V, Zakowski M, Doherty J, Politi K, Sarkaria I, Singh B, Heelan R, Rusch V, Fulton L, et al: EGF receptor gene mutations are common in lung cancers from 'never smokers' and are associated with sensitivity of tumors to gefitinib and erlotinib. Proc Natl Acad Sci USA 101: 13306-13311, 2004

12. Landi MT, Dracheva T, Rotunno M, et al: Gene expression signature of cigarette smoking and its role in lung adenocarcinoma development and survival. PLoS One 3: e1651, 2008.

13. Wu J, Irizarry R, MacDonald J and Gentry J. RIwcfJMJ. gcrma: Background Adjustment Using Sequence Information. $\mathrm{R}$ package version 2.38.0

14. Ritchie ME, Phipson B, Wu D, Hu Y, Law CW, Shi W and Smyth GK: limma powers differential expression analyses for RNA-sequencing and microarray studies. Nucleic Acids Res: January 20,2015 (Epub ahead of print). doi: 10.1093/nar/gkv007.

15. Shannon P, Markiel A, Ozier O, Baliga NS, Wang JT, Ramage D, Amin N, Schwikowski B, Ideker T. Cytoscape: A software environment for integrated models of biomolecular interaction networks. Genome Res 13: 2498-2504, 2013

16. Livak KJ and Schmittgen TD: Analysis of relative gene expression data using real-time quantitative PCR and the 2 (-Delta Delta C (T)) Method. Methods 25: 402-408, 2001.

17. Warnes GR, Bolker B, Bonebakker L, Gentleman R, Liaw WHA, Lumley T, Maechler M, Magnusson A, Moeller S, Schwartz M and Venables B: gplots: Various R Programming Tools for Plotting Data. $\mathrm{R}$ package version 2.16.0.

18. Carlson M: GO.db: A set of annotation maps describing the entire Gene Ontology. R package version 3.0.0.

19. Carlson M: KEGG.db: A set of annotation maps for KEGG $\mathrm{R}$ package version 3.0.0

20. Tenenbaum D: KEGGREST: Client-side REST access to KEGG. R package version 1.6.4.

21. Mo YQ, Dai L, Zheng DH, Zhu LJ, Wei XN, Pessler F, Shen J and Zhang BY: Synovial infiltration with CD79a-positive B cells, but not other B cell lineage markers, correlates with joint destruction in rheumatoid arthritis. J Rheumatol 38 : 2301-2308, 2011.

22. Han F, Gu D, Chen Q and Zhu H: Caveolin-1 acts as a tumor suppressor by down-regulating epidermal growth factor receptor-mitogen-activated protein kinase signaling pathway in pancreatic carcinoma cell lines. Pancreas 38: 766-774, 2009

23. Lobos-González L, Aguilar L, Diaz J, et al: E-cadherin determines Caveolin-1 tumor suppression or metastasis enhancing function in melanoma cells. Pigment Cell Melanoma Res 26: 555-570, 2013

24. Ashburner M, Ball CA, Blake JA, Botstein D, Butler H, Cherry JM, Davis AP, Dolinski K, Dwight SS, Eppig JT, et al; The Gene Ontology Consortium: Gene ontology: Tool for the unification of biology. Nat Genet 25: 25-29, 2000.

25. Kanehisa M and Goto S: KEGG: Kyoto encyclopedia of genes and genomes. Nucleic Acids Res 28: 27-30, 2000.

26. Rudin CM, Avila-Tang E, Harris CC, Herman JG, Hirsch FR, Pao W, Schwartz AG, Vahakangas KH and Samet JM: Lung cancer in never smokers: molecular profiles and therapeutic implications. Clin Cancer Res 15: 5646-5661, 2009.
27. Szymanowska-Narloch A, Jassem E, Skrzypski M, Muley T, Meister M, Dienemann H, Taron M, Rosell R, Rzepko R, Jarzab M, et al: Molecular profiles of non-small cell lung cancers in cigarette smoking and never-smoking patients. Adv Med Sci 58: 196-206, 2013.

28. Woenckhaus M, Klein-Hitpass L, Grepmeier U, Merk J, Pfeifer M, Wild P, Bettstetter M, Wuensch P, Blaszyk H, Hartmann A, et al: Smoking and cancer-related gene expression in bronchial epithelium and non-small-cell lung cancers. J Pathol 210: 192-204, 2006.

29. Spira A, Beane JE, Shah V, Steiling K, Liu G, Schembri F, Gilman S, Dumas YM, Calner P, Sebastiani P, et al: Airway epithelial gene expression in the diagnostic evaluation of smokers with suspect lung cancer. Nat Med 13: 361-366, 2007.

30. Staaf J, Jönsson G, Jönsson M, Karlsson A, Isaksson S, Salomonsson A, Pettersson HM, Soller M, Ewers SB, Johansson L, et al: Relation between smoking history and gene expression profiles in lung adenocarcinomas. BMC Med Genomics 5: 22, 2012.

31. Du X, Qian X, Papageorge A, Schetter AJ, Vass WC, Liu X, Braverman R, Robles AI and Lowy DR: Functional interaction of tumor suppressor DLC1 and caveolin-1 in cancer cells. Cancer Res 72: 4405-4416, 2012.

32. Chanvorachote $\mathrm{P}$ and Chunhacha P: Caveolin-1 regulates endothelial adhesion of lung cancer cells via reactive oxygen species-dependent mechanism. PLoS One 8: e57466, 2013.

33. Wang Z, Sokolovska A, Seymour R, Sundberg JP and Hogenesch H: SHARPIN is essential for cytokine production, $\mathrm{NF}-\kappa \mathrm{B}$ signaling, and induction of Th1 differentiation by dendritic cells. PLoS One 7: e31809, 2012.

34. Pancotti F, Roncuzzi L, Maggiolini M and Gasperi-Campani A: Caveolin-1 silencing arrests the proliferation of metastatic lung cancer cells through the inhibition of STAT3 signaling. Cell Signal 24: 1390-1397, 2012.

35. Luanpitpong S, Talbott SJ, Rojanasakul Y, Nimmannit U, Pongrakhananon V, Wang L and Chanvorachote P: Regulation of lung cancer cell migration and invasion by reactive oxygen species and caveolin-1. J Biol Chem 285: 38832-38840, 2010.

36. Wang Z, Potter CS, Sundberg JP and Hogenesch H: SHARPIN is a key regulator of immune and inflammatory responses. J Cell Mol Med 16: 2271-2279, 2012.

37. Lemjabbar H, Li D, Gallup M, Sidhu S, Drori E and Basbaum C: Tobacco smoke-induced lung cell proliferation mediated by tumor necrosis factor alpha-converting enzyme and amphiregulin. J Biol Chem 278: 26202-26207, 2003.

38. Luppi F, Aarbiou J, van Wetering S, et al: Effects of cigarette smoke condensate on proliferation and wound closure of bronchial epithelial cells in vitro: Role of glutathione. Respir Res 6: 140, 2005.

39. Schaal C and Chellappan SP: Nicotine-mediated cell proliferation and tumor progression in smoking-related cancers. Mol Cancer Res 12: 14-23, 2014.

40. Liu C, Russell RM and Wang XD: Lycopene supplementation prevents smoke-induced changes in p53, p53 phosphorylation, cell proliferation, and apoptosis in the gastric mucosa of ferrets. J Nutr 136: 106-111, 2006.

41. Dasgupta P, Rastogi S, Pillai S, et al: Nicotine induces cell proliferation by beta-arrestin-mediated activation of Src and Rb-Raf-1 pathways. J Clin Invest 116: 2208-2217, 2006.

42. Potter CS, Wang Z, Silva KA, et al: Chronic proliferative dermatitis in Sharpin null mice: development of an autoinflammatory disease in the absence of B and T lymphocytes and IL4/IL13 signaling. PLoS One 9: e85666, 2014.

43. Conklin BS, Zhao W, Zhong DS and Chen C: Nicotine and cotinine up-regulate vascular endothelial growth factor expression in endothelial cells. Am J Pathol 160: 413-418, 2002.

44. Jull BA, Plummer HK III and Schuller HM: Nicotinic receptor-mediated activation by the tobacco-specific nitrosamine NNK of a Raf-1/MAP kinase pathway, resulting in phosphorylation of c-myc in human small cell lung carcinoma cells and pulmonary neuroendocrine cells. J Cancer Res Clin Oncol 127: 707-717, 2001

45. Furrukh M: Tobacco Smoking and Lung Cancer: Perception-changing facts. Sultan Qaboos Univ Med J 13: 345-358, 2013.

46. Pfeifer GP, Denissenko MF, Olivier M, et al: Tobacco smoke carcinogens, DNA damage and p53 mutations in smoking-associated cancers. Oncogene 21: 7435-7451, 2002.

47. Husgafvel-Pursiainen $\mathrm{K}$ and Kannio A: Cigarette smoking and p53 mutations in lung cancer and bladder cancer. Environ Health Perspect 104 (Suppl 3): 553-556, 1996. 
48. Brennan JA, Boyle JO, Koch WM, Goodman SN, Hruban RH, Eby YJ, Couch MJ, Forastiere AA and Sidransky D: Association between cigarette smoking and mutation of the p53 gene in squamous-cell carcinoma of the head and neck. N Engl J Med 332: 712-717, 1995.
49. Muller PA and Vousden KH: p53 mutations in cancer. Nat Cell Biol 15: 2-8, 2013.

50. Muller PA and Vousden KH: Mutant p53 in cancer: new functions and therapeutic opportunities. Cancer Cell 25: 304-317, 2014. 